NASA Contractor Report 181901

ICASE Report No. 89-50

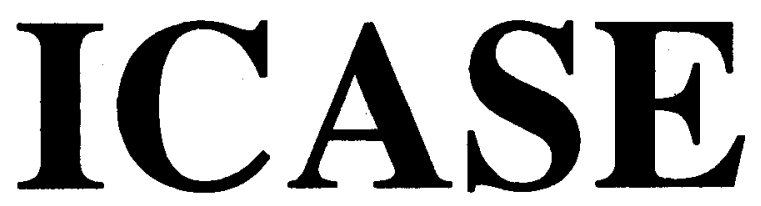

\title{
A NONLINEAR INVESTIGATION OF THE STATIONARY MODE OF INSTABILITY OF THE THREE-DIMENSIONAL COMPRESSIBLE BOUNDARY LAYER DUE TO A ROTATING DISC
}

\section{S. O. Seddougui}

Contract No. NAS1-18605

July 1989

Institute for Computer Applications in Science and Engineering NASA Langley Research Center

Hampton, Virginia 23665-5225

Operated by the Universities Space Research Association

\section{N/Sก}

National Aeronautics and

Space Administration

\section{Langley Research Center}

Lamntan Virainia 23655_5225

(NASA-CR-181901) A NONL INEAR INVESTIGATION

OF THE STATIONARY MODES OF INSTABILITY OF

$N 89-28760$

THE THREE-OIMFNSIONAL COMPRFSSIBLE ROUNCARY

LAYER DUE TO A ROTATING OISC Final Report 


\title{
INSTITUTE FOR COMPUTER APPLICATIONS \\ IN SCIENCE AND ENGINEERING
}

\author{
December 1, 1989
}

\section{SPECIAL NOTICE}

The attached figures were inadvertently omitted from ICASE Report No. 89-50, authored by Sharon Seddougui. Please insert these figures in the report that was previously mailed to you.

We apologize for any inconvenience this may have caused.

ICASE Publications Office

Reply to: ICASE, Mail Stop 132C, NASA Langley Research Center, Hampton, Virginia 23665-5225 - Telephone: 804-864-2174 OR: info@icase.edu

Fax NO. (804) 864-6134 


\title{
A NONLINEAR INVESTIGATION OF THE STATIONARY MODES OF INSTABILITY OF THE THREE-DIMENSIONAL COMPRESSIBLE BOUNDARY LAYER DUE TO A ROTATING DISC
}

\author{
Sharon O. Seddougui ${ }^{1}$ \\ Institute for Computer Applications in Science and Engineering \\ NASA Langley Research Center \\ Hampton, VA 23665
}

\begin{abstract}
This work investigates the effects of compressibility on a stationary mode of instability of the three-dimensional bouncia y layer due to a rotating disc. The aim is to determine whether this mode will be important in the finite amplitude destabilization of the boundary layer. This stationary mode is characterized by the effective velocity profile having zero shear stress at the wall. Triple-deck solutions are presented for an adiabatic wall and an isothermal wall. It is found that this stationary mode is only possible over a finite range of Mach numbers. Asymptotic solutions are obtained which describe the structure of the wavenumber and the orientation of these modes as functions of the local Mach number. The effects of nonlinearity are investigated allowing the finite amplitude growth of a disturbance close to the neutral location to be described. The results are compared with the incompressible results of $P$. Hall (Proc. R. Soc. Lond. A406, 93-106 (1986)) and S. O. MacKerrell (Proc. R. Soc. Lond. A413, 497-513 (1987)).
\end{abstract}

\footnotetext{
${ }^{1}$ This research was supported by the National Aeronautics and Space Administration under NASA Contract No. NAS1-18605 while the author was in residence at the Institute for Computer Applications in Science and Engineering (ICASE), NASA Langley Research Center, Hampton, VA 23665.
} 


\section{INTRODUCTION}

The problem considered here is that of the instability of the compressible boundary layer on a rotating disc. The flow due to a rotating disc has a three-dimensional boundary layer and exhibits a crossflow instability. This boundary layer is similar to that which occurs on swept wings and so can be used to predict the possible modes of instability of boundary layers on swept wings. Interest in these types of instabilities has increased recently due to the development of laminar flow wings. Our aim here is to investigate the effects of compressibility on a mode of instability which will be described below. For incompressible three-dimensional boundary layers this particular mode of instability may play a crucial role in the finite amplitude destabilization of the boundary layer.

One of the first experimental and theoretical investigations of instabilities of threedimensional boundary layers was conducted by Gregory, Stuart and Walker (1955). Using the china clay visualization technique they observed a set of stationary vortices in the form of spiral streaks rotating with the disc. The number of vortices observed was 28-31 with the angle between the tangent at a given point on the vortex and the normal to the radius vector being $13^{\circ}$. Linear stability analysis was used by Gregory, Stuart and Walker (1955) to explain the nature of the instability. This was described as an inviscid disturbance with an effective velocity profile having zero velocity at an inflexion point. The theory presented enabled the orientation and the wavenumber of the vortices to be predicted. The orientation of the vortices predicted by this theory was found to be consistent with the experimental observations but the vortex wavenumber predicted was about four times the value observed.

Later linear stability investigations following on from that of Gregory, Stuart and Walker (1955) of the incompressible flow due to a rotating disc were given by Cebeci and Stewartson (1980), Malik, Wilkinson and Orszag (1981), Mack (1985) and Malik (1986). The results of Malik, Wilkinson and Orszag (1981) show that the effects of streamline curvature and Coriolis force may be important. (This result was first found in the context of the Ekman boundary layer, by Faller and Kaylor (1966) and Lilly (1966).) Malik (1986) obtained the finite Reynolds number continuation of the inviscid mode of Gregory, Stuart and Walker (1955) and showed that at large Reynolds numbers an additional stationary mode can occur. The structure of this mode was subsequently investigated by Hall (1986) who showed that it has a triple-deck structure and that it corresponds to an effective velocity profile having zero shear stress at the wall. Later MacKerrell (1987) investigated the nonlinear development of this stationary mode and obtained the important result that it is destabilised by nonlinearity and thus may cause the premature growth of instability waves if it is forced above a certain threshold level. 
Visible evidence that an additional stationary mode of instability may occur was given by the experiments of Fedorov et al. (1976). They observed a stationary vortex structure having 14-16 vortices with the angle between the tangent on a point of the spiral and the normal to the radius vector being $20^{\circ}$. Thus this structure has a much lower wavenumber than that of the Gregory, Stuart and Walker mode and also a different wave angle.

The main aim of the present paper is to see how the stationary mode of Hall (1986) develops into the compressible regime. Most importantly we want to see how far into the compressible regime the potentially important subcritical nature of the instability persists. We show that the mode of instability is in fact possible only over a finite range of Mach numbers.

In Section 2, we give the full compressible equations governing the flow of interest and the boundary conditions to be satisfied. These equations are solved in Section 3 for large Reynolds number using a Dorodnitsyn-Howarth transformation to give the basic threedimensional compressible flow due to a rotating disc. We consider two cases of an adiabatic disc and an isothermal disc. In Section 4, this basic flow is perturbed by adding a small three-dimensional disturbance to it and a nonlinear stability analysis is presented. The triple-deck structure of the disturbed flow is described. Although the triple-deck scalings here differ from those of the classic triple-deck the same principles apply. A solvability condition on the linear lower deck problem produces an eigenrelation, the solutions of which are discussed. In order to obtain an equation for the disturbance amplitude we perturb the solution a small distance from the neutral location. A solvability condition in the lower deck problem produces an equation for the disturbance amplitude, the solution of which is discussed. Finally, in Section 5 the effects of compressibility are discussed and the results are compared to those obtained for an incompressible flow by Hall (1986) and MacKerrell (1987).

\section{THE GOVERNING EQUATIONS}

We will consider the steady flow of a compressible viscous fluid above an infinite flat disc rotating with an angular velocity $\Omega$ about the $z$-axis. Thus, the fluid motion is induced by the rotation of the disc and the effects of streamline curvature and Coriolis force must be taken into account. The problem is formulated in terms of cylindrical polar coordinates to take advantage of the axisymmetric motion which occurs in the basic flow. Thus, we introduce the non-dimensional coordinates and velocities $(r, \theta, z)$ and $\left(v_{r}, v_{\theta}, v_{z}\right)$, with the $z$-axis being normal to the surface of the disc. The lengths $r$ and $z$ and the velocities have been made dimensionless with respect to some reference length $\ell$ and $\Omega \ell$, respectively.

Since the fluid is compressible the variation of the density of the fluid must be consid- 
ered. Hence, the Navier-Stokes equations and the continuity equation are not sufficient to solve the problem. Thus, we must also have an equation of state and an energy equation. The Reynold number for the flow is defined by

$$
R=\frac{\Omega \ell^{2} \bar{\rho}_{\infty}}{\bar{\mu}_{\infty}},
$$

where $\bar{\rho}_{\infty}$ and $\bar{\mu}_{\infty}$ are the free-stream values of the density and the shear viscosity of the fluid, respectively. The Reynolds number is taken to be large in the following analysis. The density and temperature of the fluid are non-dimensionalized with respect to their free stream values, while the shear viscosity and the bulk viscosity are both non-dimensionalized with respect to the free-stream value of the shear viscosity. Finally, the pressure is nondimensionalized with respect to $\bar{\rho}_{\infty} \Omega^{2} \ell^{2}$. The continuity and Navier-Stokes equation for a steady compressible fluid in a reference frame rotating with angular velocity $\Omega$ are

$$
\begin{gathered}
\frac{1}{r} \frac{\partial}{\partial r}\left(\rho r v_{r}\right)+\frac{1}{r} \frac{\partial}{\partial \theta}\left(\rho v_{\theta}\right)+\frac{\partial}{\partial z}\left(\rho v_{z}\right)=0, \\
\rho\left(v_{r} \frac{\partial v_{r}}{\partial r}+\frac{v_{\theta}}{r} \frac{\partial v_{r}}{\partial \theta}+v_{z} \frac{\partial v_{r}}{\partial z}-\frac{v_{\theta}^{2}}{r}-2 v_{\theta}-r\right)=-\frac{\partial p}{\partial r}+\frac{1}{R}\left\{\frac{\partial}{\partial r}\left[2 \mu \frac{\partial v_{r}}{\partial r}+\left(\mu^{\prime}-\frac{2 \mu}{3}\right) \nabla \cdot \underline{v}\right]\right. \\
\left.+\frac{1}{r} \frac{\partial}{\partial \theta}\left[\mu\left(\frac{1}{r} \frac{\partial v_{r}}{\partial \theta}+\frac{\partial v_{\theta}}{\partial r}-\frac{v_{\theta}}{r}\right)\right]+\frac{\partial}{\partial z}\left[\mu\left(\frac{\partial v_{r}}{\partial z}+\frac{\partial v_{z}}{\partial r}\right)\right]+\frac{2 \mu}{r}\left(\frac{\partial v_{r}}{\partial r}-\frac{1}{r} \frac{\partial v_{\theta}}{\partial \theta}-\frac{v_{r}}{r}\right)\right\}, \\
\rho\left(v_{r} \frac{\partial v_{\theta}}{\partial r}+\frac{v_{\theta}}{r} \frac{\partial v_{\theta}}{\partial \theta}+v_{z} \frac{\partial v_{\theta}}{\partial z}+\frac{v_{r} v_{\theta}}{r}+2 v_{r}\right)=-\frac{1}{r} \frac{\partial p}{\partial \theta}+\frac{1}{R}\left\{\frac{1}{r} \frac{\partial}{\partial \theta}\left[2 \mu \frac{\partial v_{\theta}}{\partial \theta}+\left(\mu^{\prime}-\frac{2 \mu}{3}\right) \nabla \cdot \underline{v}\right]\right. \\
\left.+\frac{\partial}{\partial z}\left[\mu\left(\frac{1}{r} \frac{\partial v_{z}}{\partial \theta}+\frac{\partial v_{\theta}}{\partial z}\right)\right]+\frac{\partial}{\partial r}\left[\mu\left(\frac{1}{r} \frac{\partial v_{r}}{\partial \theta}+\frac{\partial v_{\theta}}{\partial r}-\frac{v_{\theta}}{r}\right)\right]+\frac{2 \mu}{r}\left(\frac{1}{r} \frac{\partial v_{r}}{\partial \theta}+\frac{\partial v_{\theta}}{\partial r}-\frac{v_{\theta}}{r}\right)\right\}, \\
\rho\left(v_{r} \frac{\partial v_{z}}{\partial r}+\frac{v_{\theta}}{r} \frac{\partial v_{z}}{\partial \theta}+v_{z} \frac{\partial v_{z}}{\partial z}\right)=-\frac{\partial p}{\partial z}+\frac{1}{R}\left\{\frac{\partial}{\partial z}\left[2 \mu \frac{\partial v_{z}}{\partial z}+\left(\mu^{\prime}-\frac{2 \mu}{3}\right) \nabla \cdot, \underline{v}\right]\right. \\
\left.+\frac{1}{r} \frac{\partial}{\partial r}\left[\mu r\left(\frac{\partial v_{r}}{\partial z}+\frac{\partial v_{z}}{\partial r}\right)\right]+\frac{1}{r} \frac{\partial}{\partial \theta}\left[\mu\left(\frac{1}{r} \frac{\partial v_{z}}{\partial \theta}+\frac{\partial v_{\theta}}{\partial z}\right)\right]\right\} .
\end{gathered}
$$

Here $\rho$ is the density, $p$ is the fluid pressure, $\mu$ and $\mu^{\prime}$ are the shear and bulk viscosities, respectively and $\nabla \cdot \underline{v}$ is defined by

$$
\nabla \cdot \underline{v}=\frac{1}{r} \frac{\partial}{\partial r}\left(r v_{r}\right)+\frac{1}{r} \frac{\partial v_{\theta}}{\partial \theta}+\frac{\partial v_{z}}{\partial z} .
$$

We consider the fluid to be a perfect gas then the equation of state is

$$
p=\frac{1}{\gamma M_{\infty}^{2}} \rho T,
$$


where $T$ denotes the temperature of the fluid. The ratio of specific heats of the fluid is defined by

$$
\gamma=\frac{c_{p}}{c_{v}},
$$

where $c_{p}$ is the specific heat at constant pressure and $c_{v}$ is the specific heat at constant volume. The free-stream Mach number is defined by

$$
M_{\infty}=\frac{\Omega \ell}{\sqrt{\gamma R \bar{T}_{\infty}}},
$$

where $\bar{T}_{\infty}$ is the free-stream temperature and $R$ is the gas constant, which can be expressed as $R=c_{p}-c_{v}$. Using (2.3) the energy equation for a steady flow may be written in terms of the temperature and is given by

$$
\begin{gathered}
\frac{\rho}{(\gamma-1) M_{\infty}^{2}}\left(v_{r} \frac{\partial T}{\partial r}+\frac{v_{\theta}}{r} \frac{\partial T}{\partial \theta}+v_{z} \frac{\partial T}{\partial z}\right)=v_{r} \frac{\partial p}{\partial r}+\frac{v_{\theta}}{r} \frac{\partial p}{\partial \theta}+v_{z} \frac{\partial p}{\partial z}+\frac{\Phi}{R} \\
+\frac{1}{R(\gamma-1) M_{\infty}^{2}}\left[\frac{1}{r} \frac{\partial}{\partial r}\left(r k \frac{\partial T}{\partial r}\right)+\frac{1}{r^{2}} \frac{\partial}{\partial \theta}\left(k \frac{\partial T}{\partial \theta}\right)+\frac{\partial}{\partial z}\left(k \frac{\partial T}{\partial z}\right)\right],
\end{gathered}
$$

where $k$ is the coefficient of thermal diffusivity and has been non-dimensionalized with respect to $\bar{\mu}_{\infty} c_{p}$. This is related to the Prandtl number $\sigma$ by

$$
\sigma=\frac{\mu}{k} .
$$

The dissipation function $\Phi$ in (2.4a) is given by

$$
\begin{aligned}
\Phi= & 2 \mu\left[\left(\frac{\partial v_{r}}{\partial r}\right)^{2}+\left(\frac{1}{r} \frac{\partial v_{\theta}}{\partial \theta}+\frac{v_{r}}{r}\right)^{2}+\left(\frac{\partial v_{z}}{\partial z}\right)^{2}+\frac{1}{2}\left(\frac{\partial v_{z}}{\partial \theta}+\frac{\partial v_{\theta}}{\partial z}\right)^{2}+\frac{1}{2}\left(\frac{\partial v_{r}}{\partial z}+\frac{\partial v_{z}}{\partial r}\right)^{2}\right. \\
& \left.+\frac{1}{2}\left(\frac{1}{r} \frac{\partial v_{r}}{\partial \theta}+\frac{\partial v_{\theta}}{\partial r}-\frac{v_{\theta}}{r}\right)^{2}\right]+\left(\mu^{\prime}-\frac{2 \mu}{3}\right)\left(\frac{\partial v_{r}}{\partial r}+\frac{1}{r} \frac{\partial v_{\theta}}{\partial \theta}+\frac{v_{r}}{r}+\frac{\partial v_{z}}{\partial z}\right)^{2} .
\end{aligned}
$$

The boundary conditions for the flow are that the no-slip conditions must be satisfied at the surface of the disc and there should be no motion far away from the disc. Hence, we have

$$
v_{r}=v_{\theta}=v_{z}=0 \text { at } z=0,
$$

and

$$
v_{r} \rightarrow 0 \text { and } v_{\theta} \rightarrow-r \text { as } z \rightarrow \infty \text {. }
$$

The density, temperature, and viscosities must approach their free-stream values as $z \rightarrow \infty$, which, since they are non-dimensionalized with respect to these values, are equal to unity. We consider two possibilities for the boundary condition on the temperature at the surface of the disc. Either, the surface is adiabatic (i.e. thermally insulated) and then

$$
\frac{\partial T}{\partial z}=0 \text { at } z=0
$$


or the surface is isothermal (i.e. the temperature of the surface is prescribed). In this case we suppose that the surface temperature is $T_{B W}$ then the boundary condition is

$$
T=T_{B W} \text { at } z=0
$$

\section{THE BASIC FLOW}

We now consider the three-dimensional solution of equations (2.2) - (2.5) for the steady compressible boundary layer flow over a rotating disc, for which the Reynolds number will be large. We define the non-dimensional coordinates

$$
(r, \theta, z)=\left(r, \theta, R^{-\frac{1}{2}} z_{B}\right)
$$

and velocities

$$
\left(v_{r}, v_{\theta}, v_{z}\right)=\underline{V}_{B}=\left(u_{B}, v_{B}, R^{-\frac{1}{2}} w_{B}\right)
$$

with the pressure, density, shear viscosity, bulk viscosity, conductivity, and temperature denoted by $p_{B}, \rho_{B}, \mu_{B}, \mu_{B}^{\prime}, k_{B}, T_{B}$, respectively. This flow will be defined by the governing equations (2.2) - (2.5) with $\partial / \partial \theta \equiv 0$, since the flow will be axisymmetric. We substitute the basic flow variables (3.1) into the governing equations and neglect terms of $0\left(R^{-1}\right)$ in the Navier-Stokes equations and $0\left(R^{-\frac{1}{2}}\right)$ in the energy equation. Hence, for large Reynolds number the basic flow quantities are determined from the following equations:

$$
\begin{gathered}
\rho_{B}\left(\frac{\partial u_{B}}{\partial r}+\frac{\partial w_{B}}{\partial z_{B}}\right)+u_{B} \frac{\partial \rho_{B}}{\partial r}+w_{B} \frac{\partial \rho_{B}}{\partial z_{B}}+\frac{\rho_{B} u_{B}}{r}=0, \\
\rho_{B}\left(u_{B} \frac{\partial u_{B}}{\partial r}+w_{B} \frac{\partial u_{B}}{\partial z_{B}}-\frac{v_{B}^{2}}{r}-2 v_{B}-r\right)=-\frac{\partial p_{B}}{\partial r}+\frac{\partial}{\partial z_{B}}\left(\mu_{B} \frac{\partial u_{B}}{\partial z_{B}}\right), \\
\rho_{B}\left(u_{B} \frac{\partial v_{B}}{\partial r}+w_{B} \frac{\partial v_{B}}{\partial z_{B}}+\frac{u_{B} v_{B}}{r}+2 u_{B}\right)=\frac{\partial}{\partial z_{B}}\left(\mu_{B} \frac{\partial v_{B}}{\partial z_{B}}\right), \\
\frac{\partial p_{B}}{\partial z_{B}}=0, \\
p_{B}=\frac{1}{\gamma M_{\infty}^{2}} \rho_{B} T_{B}, \\
\left.\frac{\partial T_{B}}{\partial r}+w_{B} \frac{\partial T_{B}}{\partial z_{B}}\right)=u_{B} \frac{\partial p_{B}}{\partial r}+w_{B} \frac{\partial p_{B}}{\partial z_{B}}+\mu_{B}\left[\left(\frac{\partial u_{B}}{\partial z_{B}}\right)^{2}+\left(\frac{\partial v_{B}}{\partial z_{B}}\right)^{2}\right] \\
+\frac{1}{(\gamma-1) M_{\infty}^{2}}\left(u_{B}^{2} \frac{\partial}{\partial z_{B}}\left(k_{B} \frac{\partial T_{B}}{\partial z_{B}}\right) .\right.
\end{gathered}
$$

The boundary conditions are

$$
u_{B}=v_{B}=w_{B}=0 \text { at } z_{B}=0
$$




$$
\begin{gathered}
u_{B} \rightarrow 0, v_{B} \rightarrow-r \text { as } z_{B} \rightarrow \infty, \\
\rho_{B}, T_{B}, \mu_{B} \rightarrow 1, p_{B} \rightarrow \frac{1}{\gamma M_{\infty}^{2}} \text { as } z_{B} \rightarrow \infty .
\end{gathered}
$$

Since we require some properties of the basic flow in the later stability analysis we proceed to find the solution of (3.2) and (3.3). For an incompressible flow the basic flow can be given by Kármán's steady solution (see Kármán's (1921)).

It is found that the present problem can be reduced to the corresponding incompressible one by use of the Dorodnitsyn - Howarth transformation which will be described below. (Riley (1964) obtains the same results using the Von Mises transformation.) Since the fluid is stationary everywhere outside the boundary layer the pressure gradient $\partial p_{B} / \partial r$ will be zero everywhere. Thus, from $(3.2 \mathrm{~d}, \mathrm{e})$ and the boundary conditions $p_{B}$ is constant and equal to $\left(\gamma M_{\infty}^{2}\right)^{-1}$. We assume the Chapman viscosity law

$$
\mu=C T,
$$

where $\mathrm{C}$ is a constant, then the energy equation is decoupled from the momentum equations. We take $C=\mu_{B W} / T_{B W}$, where the subscripts $\mathrm{w}$ indicate values at the wall, then the law (3.4a) will be most realistic close to the wall. We use Sutherland's law to evaluate $\mu_{B W}$, hence

$$
\mu_{B W}=T_{B W}^{3 / 2}\left(\frac{1+C_{1} / \bar{T} \infty}{T_{B W}+C_{1} / \bar{T} \infty}\right),
$$

where the constant $C_{1}$ is given by $C_{1}=198.6^{\circ} \mathrm{R}$. Since the pressure is constant from (3.2e) $\rho_{B} T_{B}$ is constant and so the Chapman viscosity law (3.4a) gives $\rho_{B} \mu_{B}$ is constant. From the boundary conditions as $z_{B} \rightarrow \infty$ we find that $\rho_{B} \mu_{B}=C$. It is as a result of this expression that the energy equation is decoupled from the momentum equations. Thus, we have

$$
\mu_{B}=\left(\frac{\mu_{B W}}{T_{B W}}\right) T_{B}
$$

and

$$
\rho_{B}=\frac{1}{T_{B}}
$$

We make the Dorodnitsyn - Howarth transformation (see for example Stewartson (1964)) and introduce the coordinate

$$
\eta=\frac{1}{C^{1 / 2}} \int_{0}^{z_{B}} \rho_{B} d z_{B},
$$

and the stream function $\psi$, where

$$
u_{B}=\frac{1}{r} \frac{\partial \psi}{\partial \eta} \text { and } w_{B}=-\frac{1}{r \rho_{B}} \frac{\partial \psi}{\partial r},
$$


where $\partial / \partial r$ denotes a derivative with $\psi$ held constant. Then the equations (3.2) become

$$
\begin{gathered}
\frac{1}{r} \frac{\partial \psi}{\partial \eta} \frac{\partial}{\partial r}\left(\frac{1}{r} \frac{\partial \psi}{\partial \eta}\right)-\frac{1}{r} \frac{\partial \psi}{\partial r} \frac{\partial}{\partial \eta}\left(\frac{1}{r} \frac{\partial \psi}{\partial \eta}\right)-\frac{v_{B}^{2}}{r}-2 v_{B}-r=\frac{\partial^{2}}{\partial \eta^{2}}\left(\frac{1}{r} \frac{\partial \psi}{\partial \eta}\right), \\
\frac{1}{r} \frac{\partial \psi}{\partial \eta} \frac{\partial v_{B}}{\partial r}-\frac{1}{r} \frac{\partial \psi}{\partial r} \frac{\partial v_{B}}{\partial \eta}+\frac{1}{r^{2}} \frac{\partial \psi}{\partial \eta} v_{B}+\frac{2}{r} \frac{\partial \psi}{\partial \eta}=\frac{\partial^{2} v_{B}}{\partial \eta^{2}}, \\
\frac{1}{(\gamma-1) M_{\infty}^{2}}\left(\frac{1}{r} \frac{\partial \psi}{\partial \eta} \frac{\partial T_{B}}{\partial r}-\frac{1}{r} \frac{\partial \psi}{\partial r} \frac{\partial T_{B}}{\partial \eta}\right)=\left[\left(\frac{\partial}{\partial \eta}\left(\frac{1}{r} \frac{\partial \psi}{\partial \eta}\right)\right)^{2}+\left(\frac{\partial v_{B}}{\partial \eta}\right)^{2}\right]+\frac{1}{\sigma(\gamma-1) M_{\infty}^{2}} \frac{\partial^{2} T_{B}}{\partial \eta^{2}} .
\end{gathered}
$$

Now if we define $\psi$ by

then from $(3.6 \mathrm{~b}, \mathrm{c})$

$$
\psi=-\frac{r^{2}}{2} H(\eta)
$$

$$
\begin{aligned}
u_{B} & =-\frac{r}{2} H^{\prime}(\eta), \\
w_{B} & =\frac{C^{1 / 2}}{\rho_{B}} H(\eta),
\end{aligned}
$$

where a prime denotes differentiation with respect to $\eta$. We also define the function $G(\eta)$ by

$$
v_{B}=r G(\eta) .
$$

Hence, using (3.8) from (3.7a,b) we find that the functions $H$ and $G$ satisfy

$$
\begin{gathered}
H^{\prime \prime \prime}+\frac{\left(H^{\prime}\right)^{2}}{2}-H H^{\prime \prime}-2(G+1)^{2}=0, \\
G^{\prime \prime}+H^{\prime}(G+1)-H G^{\prime}=0, \\
H^{\prime}(0)=G(0)=0, \\
H^{\prime}(\infty)=0, G(\infty)=-1 .
\end{gathered}
$$

These are the equations satisfied by Kármán's steady solution which may be solved numerically. Two results from the solutions of (3.9) that we require in the later analysis are

$$
\bar{u}_{0}=\frac{-H^{\prime \prime}(0)}{2}=0.51023 \text { and } v_{0}=G^{\prime}(0)=-0.61592 .
$$

The energy equation (3.7c) becomes

$$
\frac{1}{(\gamma-1) M_{\infty}^{2}}\left(-\frac{r H^{\prime}}{2} \frac{\partial T_{B}}{\partial r}+H T_{B}^{\prime}\right)=\frac{r^{2}}{4}\left(H^{\prime \prime}\right)^{2}+r^{2}\left(G^{\prime}\right)^{2}+\frac{T_{B}^{\prime \prime}}{\sigma(\gamma-1) M_{\infty}^{2}} .
$$

The solution to this equation can be found in terms of a heat conducting term and a viscous dissipation term. (See, for example, Riley (1964)). The solution can be written as

$$
T_{B}=1+\left(T_{B W}-1\right) \phi_{1}(\eta)+(\gamma-1) \frac{M_{\infty}^{2} r^{2}}{4} \phi_{2}(\eta),
$$


where the functions $\phi_{1}$ and $\phi_{2}$ satisfy

$$
\begin{gathered}
\phi_{1}^{\prime \prime}-\sigma H \phi_{1}^{\prime}=0 \\
\phi_{2}^{\prime \prime}-\sigma H \phi_{2}^{\prime}+\sigma H^{\prime} \phi_{2}=-\sigma\left(\left(H^{\prime \prime}\right)^{2}+4\left(G^{\prime}\right)^{2}\right) .
\end{gathered}
$$

For the special case of a model fluid with $\sigma=1$, exact solutions of $\phi_{2}$ can be obtained. Asymptotic solutions are given by Riley (1964) for large and small values of $\sigma$. Note that if $\sigma$ is large the thermal boundary layer is much thinner than the viscous boundary layer and conversely, if $\sigma$ is small the thermal boundary layer is much thicker than the viscous boundary layer. In the analysis to follow we will consider $\sigma=0.72$, which is the value for air. For an adiabatic plate the boundary conditions are

$$
\begin{gathered}
\phi_{1}^{\prime}(0)=\phi_{2}^{\prime}(0)=0, \\
\phi_{1}(\infty)=\phi_{2}(\infty)=0 .
\end{gathered}
$$

Then, we find that the solution of (3.11) is $\phi_{1} \equiv 0$. For $\sigma=1$, the solution of (3.12) is given by

$$
\phi_{2}=2-\frac{\left(H^{\prime}\right)^{2}}{2}-2(G)^{2},
$$

and if $\sigma=0.72$, numerically we find that $\phi_{2}(0)=1.7899$. While, if there is heat transfer at the wall then the boundary conditions are

$$
\begin{aligned}
& \phi_{1}(0)=1, \phi_{2}(0)=0 \\
& \phi_{1}(\infty)=\phi_{2}(\infty)=0
\end{aligned}
$$

In this case, the solution of (3.11) is given by

$$
\phi_{1}(\eta)=A \int_{\eta}^{\infty} e^{-\sigma I} d \eta
$$

where

$$
\begin{gathered}
I(\eta)=-\int_{0}^{\eta} H(\eta) d \eta, \\
A^{-1}=\int_{0}^{\infty} e^{-\sigma I} d \eta .
\end{gathered}
$$

For $\sigma=0.72, A$ is found numerically to be $A=0.3286$. The solution of (3.12) has to be obtained numerically, except when $\sigma=1$ which gives

$$
\phi_{2}=-2\left(\frac{\left(H^{\prime}\right)^{2}}{4}+G(G+1)\right),
$$

and for $\sigma=0.72$ we find numerically that $\phi_{2}^{\prime}(0)=0.9348$. We will require the results for $\phi_{2}(0), \phi_{2}^{\prime}(0)$ and $A$ in the later analysis. 


\section{THE WEAKLY NONLINEAR STABILITY ANALYSIS}

We consider the lower branch stationary viscous modes described by Hall (1986) and follow the analysis of MacKerrell (1987). We perturb the basic flow described in Section 3. The disturbance will have a triple-deck structure identical to that described by Hall (1986) for incompressible flow over a rotating disc. This will be based on the small parameter $\varepsilon$, which is defined by

$$
\varepsilon=R^{-\frac{1}{16}} \text {. }
$$

The upper, main, and lower decks are of thickness of $0\left(\varepsilon^{4}\right), 0\left(\varepsilon^{8}\right)$ and $0\left(\varepsilon^{9}\right)$, respectively. The structure of the disturbances in the main and upper decks is essentially the same as that found by Smith (1979), who investigated lower-branch disturbances to Blasius flow. It is found that the viscous effects are only important in the lower deck. The upper deck flow structure is essentially inviscid and irrotational and provides a pressure gradient which drives the lower deck. In the main deck the flow structure is inviscid with no variation in pressure across the layer. The lower deck is a thin viscous layer which is required to satisfy the no-slip boundary conditions at the disc surface. The disturbance is found to have wavenumbers in the radial and azimuthal directions of $0\left(\epsilon^{4}\right)$. Thus, we seek stationary modes of instability with three-dimensional solutions proportional to

$$
E=\exp \left(\frac{i}{\varepsilon^{4}}\left[\int^{r} \alpha(r) d r+\beta \theta\right]\right) .
$$

The wavenumbers $\alpha$ and $\beta$ expand as

$$
\begin{aligned}
& \alpha=\alpha_{0}+\varepsilon^{2} \alpha_{1}+\varepsilon^{3} \alpha_{2}+\cdots, \\
& \beta=\beta_{0}+\varepsilon^{2} \beta_{1}+\varepsilon^{3} \beta_{2}+\cdots,
\end{aligned}
$$

as in the corresponding incompressible problem. Hence, we add disturbances proportional to $E$ to the basic flow described in Section 3. The forms of the expansions of the disturbance will be different in each deck and are essentially fixed by balancing the convection and viscous terms in the radial momentum equation in the lower deck.

We define the perturbed velocities, pressure, density and temperature by

$$
\begin{gathered}
\left(v_{r}, v_{\theta}, v_{z}\right)=\underline{V}_{B}+(U, V, W), \\
(p, \rho, T)=\left(p_{B}, \rho_{B}, T_{B}\right)+\left(P, \rho_{p}, T_{p}\right),
\end{gathered}
$$

where the perturbation quantities are assumed to be small. The perturbation terms will be in the form of fundamental terms and harmonic terms arising from the nonlinear nature of the governing equations. Initially, we are seeking the solution at the position of neutral 
stability so $\alpha$ and $\beta$ will be real. Later we will consider the solution close to the position of neutral stability and then $\alpha$ will be complex. We begin by obtaining the solution in the upper deck.

\subsection{The Upper-Deck Solutions}

We introduce the coordinate $Z$ where $z=\varepsilon^{4} Z$ so that $Z$ is $0(1)$ in the upper deck. The radial velocity perturbation expands as

$$
\begin{aligned}
U= & \delta\left(\varepsilon^{3} \bar{U}_{0}+\varepsilon^{4} \bar{U}_{1}+\cdots\right) E+\delta^{2}\left(\bar{U}_{20}+\varepsilon \bar{U}_{21}+\cdots\right) E^{2}+\delta^{3}\left(\frac{\bar{U}_{30}}{\varepsilon^{3}}+\frac{\bar{U}_{31}}{\varepsilon^{2}}+\cdots\right) E^{3} \\
& +\delta^{3}\left(\frac{\bar{U}_{10}}{\varepsilon^{3}}+\frac{\bar{U}_{11}}{\varepsilon^{2}}+\cdots\right) E+0\left(\delta^{4}\right)+c . c .
\end{aligned}
$$

where c.c denotes complex conjugate of the preceding expression and $\delta$ is a small amplitude which we shall later relate to $\varepsilon$. The functions $\bar{U}_{0}, \bar{U}_{20}$ etc. depend on $r$ and $Z$. The azimuthal velocity, density, and temperature perturbations have similar forms to (4.2a). Note that there are no mean flow terms, i.e., terms independent of $r$ and $\theta$.

The vertical velocity and pressure perturbations have mean flow terms and are given by

$$
\begin{aligned}
W= & \delta\left(\varepsilon^{3} \bar{W}_{0}+\varepsilon^{4} \bar{W}_{1}+\cdots\right) E+\delta^{2}\left(\bar{W}_{20}+\varepsilon \bar{W}_{21}+\cdots\right) E^{2}+\delta^{2}\left(\varepsilon^{5} \bar{W}_{m 0}+\varepsilon^{6} \bar{W}_{m 1}+\cdots\right) \\
& +\delta^{3}\left(\frac{\bar{W}_{30}}{\varepsilon^{3}}+\frac{\bar{W}_{31}}{\varepsilon^{2}}+\cdots\right) E^{3}+\delta^{3}\left(\frac{\bar{W}_{10}}{\varepsilon^{3}}+\frac{\bar{W}_{11}}{\varepsilon^{2}}+\cdots\right) E+0\left(\delta^{4}\right)+c . c . \\
P= & \delta\left(\varepsilon^{3} \bar{P}_{0}+\varepsilon^{4} \bar{P}_{1}+\cdots\right) E+\delta^{2}\left(\bar{P}_{20}+\varepsilon \bar{P}_{21}+\cdots\right) E^{2}+\delta^{2}\left(\varepsilon^{6} \bar{P}_{m 0}+\varepsilon^{7} \bar{P}_{m 1}+\cdots\right) \\
& +\delta^{3}\left(\frac{\bar{P}_{30}}{\varepsilon^{3}}+\frac{\bar{P}_{31}}{\varepsilon^{2}}+\cdots\right) E^{3}+\delta^{3}\left(\frac{\bar{P}_{10}}{\varepsilon^{3}}+\frac{\bar{P}_{11}}{\varepsilon^{2}}+\cdots\right) E+0\left(\delta^{4}\right)+c . c .
\end{aligned}
$$

Using the Chapman viscosity law (3.4) the shear viscosity, bulk viscosity and thermal conductivity perturbations have the following form:

$$
\mu_{p}=\frac{\mu_{B W}}{T_{B W}} T_{p}, \mu_{p}^{\prime}=\frac{\mu_{B W}^{\prime}}{T_{B W}} T_{p}, k_{p}=\frac{1}{\sigma} \frac{\mu_{B W}}{T_{B W}} T_{p} .
$$

From the solution for the basic flow described in Section 3, in the upper deck $u_{B}=0, v_{B}=$ $-r, \rho_{B}=1, T_{B}=1$ and $p_{B}$ is constant. We substitute the perturbed flow (4.1) with the perturbed terms given by (4.2) into the governing equations (2.2)-(2.5). We first consider the linear solutions and equate terms proportional to $\delta E$ in the resulting equations. It is 
found that the solution for the first order terms, which decays to zero as $Z \rightarrow \infty$, is given by

$$
\begin{aligned}
& \bar{U}_{0}=\frac{\alpha_{0}}{\beta_{0}} f e^{-\Gamma Z}, \quad \bar{V}_{0}=\frac{f}{r} e^{-\Gamma Z}, \quad \bar{W}_{0}=\frac{\Gamma}{\beta_{0}} f e^{-\Gamma Z}, \\
& \bar{P}_{0}=f e^{-\Gamma Z}, \quad \bar{\rho}_{0}=M_{\infty}^{2} f e^{-\Gamma Z}, \quad \bar{T}_{0}=(\gamma-1) M_{\infty}^{2} f e^{-\Gamma Z} .
\end{aligned}
$$

Here $f(r)$ is an amplitude function to be determined and

$$
\Gamma^{2}=\alpha_{0}^{2}+\frac{\beta_{0}^{2}}{r^{2}}\left(1-M_{r}^{2}\right),
$$

where $M_{r}$ is a local Mach number defined by $M_{r}=r M_{\infty}$. Hence, from (4.4), for threedimensional modes to exist for neutral wavenumbers we must have

$$
\alpha_{0}^{2}+\frac{\beta_{0}^{2}}{r^{2}}\left(1-M_{r}^{2}\right)>0 .
$$

Thus, for $\left|M_{\mathrm{r}}\right|<1$ there will exist three-dimensional solutions for all real values of $\alpha_{0}$ and $\beta_{0}$. However, for $M_{r}>1$ three-dimensional modes will only exist for $\Gamma^{2}>0$. We shall see later that from Hall (1986), in order for the three-dimensional stationary modes to exist the effective wall shear must vanish. Thus, we choose to look for stationary modes where the effective wall shear vanishes at zeroth order. The effective wall shear at zeroth order is

$$
\alpha_{0} \frac{\partial u_{B}}{\partial z_{B}}+\frac{\beta_{0}}{r} \frac{\partial v_{B}}{\partial z_{B}}=0 \text { at } z_{B}=0 .
$$

Hence, from the basic solution described in Section 3 the condition (4.6a) is

$$
\frac{\alpha_{0} r}{\beta_{0}}=-\frac{\bar{v}_{0}}{\bar{u}_{0}} \approx 1.207,
$$

where $\bar{u}_{0}$ and $\bar{v}_{0}$ are defined by (3.9e) and the solution is neutral at the position $r$ where (4.6) holds. This is the same result found by Hall (1986) for the incompressible problem. The conditions (4.5) and (4.6) imply that for the stationary three-dimensional modes of instability we are seeking to exist

$$
\left|M_{r}\right|<1.567 \text {. }
$$

Thus, these modes will not be present in high Mach number flows.

It is found that the next order terms in $\varepsilon$ have the same form as those given by (4.3) but with amplitude $g(r)$. This unknown amplitude is determined from matching the expansions in the upper deck with the expansions in the main deck and will be a linear function of $f(r)$. Analytic solutions can also be found for the higher order terms, but are not required in the present analysis. 
If we now consider the terms proportional to $\delta^{2} E^{2}$ in the governing equations we find that the first harmonic terms are

$$
\begin{gathered}
\bar{U}_{20}=\frac{\alpha_{0}}{\beta_{0}} f_{2} e^{-2 \Gamma Z}, \quad \bar{V}_{20}=\frac{f_{2}}{r} e^{-2 \Gamma Z}, \quad \bar{W}_{20}=\frac{i \Gamma}{\beta_{0}} f_{2} e^{-2 \Gamma Z}, \\
\bar{P}_{20}=f_{2} e^{-2 \Gamma Z}, \quad \bar{\rho}_{20}=M_{\infty}^{2} f_{2} e^{-2 \Gamma Z}, \quad \bar{T}_{20}=(\gamma-1) M_{\infty}^{2} f_{2} e^{-2 \Gamma Z} .
\end{gathered}
$$

Here $f_{2}(r)$ is an unknown amplitude function, which will later be found in terms of $f(r)$. There is no contribution from the nonlinear terms until the solution for $P_{25}$. It is found later that the harmonic terms proportional to $\delta^{3} E^{3}$ are not involved in the present analysis so we shall omit the solutions for these terms. Thus, we consider next the $0\left(\delta^{3} E\right)$ terms which arise from the nonlinear interaction of the $0\left(\delta E^{-1}\right)$ terms with the $0\left(\delta^{2} E^{2}\right)$ terms and the $0(\delta E)$ terms with the $0\left(\delta^{2} E^{0}\right)$ terms. In a similar way to the solutions given above we find that

$$
\begin{gathered}
\bar{U}_{10}=\frac{\alpha_{0}}{\beta_{0}} f_{4} e^{-\Gamma Z}, \quad \bar{V}_{10}=\frac{f_{4}}{r} e^{-\Gamma Z}, \quad \bar{W}_{10}=\frac{i \Gamma f_{4}}{\beta_{0}} e^{-\Gamma Z}, \\
\bar{P}_{10}=f_{4} e^{-\Gamma Z}, \quad \bar{\rho}_{10}=M_{\infty}^{2} f_{4} e^{-\Gamma Z}, \quad \bar{T}_{10}=(\gamma-1) M_{\infty}^{2} f_{4} e^{-\Gamma Z},
\end{gathered}
$$

where $f_{4}$ is an unknown function of $f(r)$. We note that the solutions for the velocities and pressure have the same form as those obtained for the incompressible problem by MacKerrell (1987), but where $\Gamma$ now depends on $M_{r}$ as well as on $\alpha_{0}$ and $\beta_{0}$.

The solutions in the main deck must match with those obtained in the upper deck as $Z \rightarrow 0$.

\subsection{The Main-Deck Solutions}

From section 3 the boundary layer variable is defined by $z_{B}=\varepsilon^{-8} z$, where $z_{B}$ is $0(1)$ in this layer. The non-dimensional radial velocity perturbation has the form

$$
\begin{gathered}
U=\delta\left(\frac{\hat{u}_{0}}{\varepsilon}+\hat{u}_{1}+\cdots\right) E+\delta^{2}\left(\frac{\hat{u}_{20}}{\varepsilon^{4}}+\frac{\hat{u}_{21}}{\varepsilon^{3}}+\cdots\right) E^{2}+\delta^{2}\left(\frac{\hat{u}_{m 0}}{\varepsilon^{2}}+\cdots\right) \\
+\delta^{3}\left(\frac{\hat{u}_{30}}{\varepsilon^{7}}+\frac{\hat{u}_{31}}{\varepsilon^{6}}+\cdots\right) E^{3}+\delta^{3}\left(\frac{\hat{u}_{10}}{\varepsilon^{7}}+\frac{\hat{u}_{11}}{\varepsilon^{6}}+\cdots\right) E+0\left(\delta^{4}\right)+c . c .
\end{gathered}
$$

with a similar expression for $V$. The vertical velocity perturbation has the following expansion:

$$
\begin{aligned}
W= & \delta\left(\varepsilon^{3} \hat{w}_{0}+\varepsilon^{4} \hat{w}_{1}+\cdots\right) E+\delta^{2}\left(\hat{w}_{20}+\varepsilon \hat{w}_{21}+\cdots\right) E^{2}+\delta^{2}\left(\varepsilon^{6} \hat{w}_{m 0}+\varepsilon^{7} \hat{w}_{m 1}+\cdots\right) \\
& +\delta^{3}\left(\frac{\hat{w}_{30}}{\varepsilon^{3}}+\frac{\hat{w}_{31}}{\varepsilon^{2}}+\cdots\right) E^{3}+\delta^{3}\left(\frac{\hat{w}_{10}}{\varepsilon^{3}}+\frac{\hat{w}_{11}}{\varepsilon^{2}}+\cdots\right) E+0\left(\delta^{4}\right)+c . c .
\end{aligned}
$$


with a similar expression for $P$. The density and temperature perturbations are similar to each other. The expansion for the density perturbation is given by

$$
\begin{aligned}
\rho_{p}= & \delta\left(\frac{\hat{\rho}_{0}}{\varepsilon}+\hat{\rho}_{1}+\cdots\right) E+\delta^{2}\left(\frac{\hat{\rho}_{20}}{\varepsilon^{4}}+\frac{\hat{\rho}_{21}}{\varepsilon^{3}}+\cdots\right) E^{2}+\delta^{2}\left(\frac{\hat{\rho}_{m 0}}{\varepsilon^{3}}+\frac{\hat{\rho}_{m 1}}{\varepsilon^{2}}+\cdots\right) \\
& +\delta^{3}\left(\frac{\hat{\rho}_{30}}{\varepsilon^{7}}+\frac{\hat{\rho}_{31}}{\varepsilon^{6}}+\cdots\right) E^{3}+\delta^{3}\left(\frac{\hat{\rho}_{10}}{\varepsilon^{7}}+\frac{\hat{\rho}_{11}}{\varepsilon^{6}}+\cdots\right) E+0\left(\delta^{4}\right)+c . c .
\end{aligned}
$$

We substitute the perturbed flow with the expansions (4.8) into the governing equations. Equating coefficients of the linear terms proportional to $\delta E$ gives the following solutions which match with the upper deck solutions as $z_{B} \rightarrow \infty$ and $Z \rightarrow 0$ :

$$
\begin{gathered}
\hat{u}_{0}=\frac{\Gamma f}{\beta_{0}^{2}} \frac{\partial u_{B}}{\partial z_{B}}, \quad \hat{v}_{0}=\frac{\Gamma f}{\beta_{0}^{2}} \frac{\partial v_{B}}{\partial z_{B}}, \hat{w}_{0}=-\frac{i \Gamma f}{\beta_{0}^{2}}\left(\alpha_{0} u_{B}+\frac{\beta_{0}}{r} v_{B}\right), \\
\hat{p}_{0}=f, \quad \hat{\rho}_{0}=\frac{\Gamma f}{\beta_{0}^{2}} \frac{\partial R_{0}}{\partial z_{B}}, \quad \hat{T}_{0}=\frac{\Gamma f}{\beta_{0}^{2}} \frac{\partial T_{B}}{\partial z_{B}},
\end{gathered}
$$

where $R_{0}$ denotes the density of the basic flow solution. The normal velocity $\hat{w}_{0}$ satisfies the no-slip condition at $z_{B}=0$, but $\hat{u}_{0}$ and $\hat{v}_{0}$ do not. As mentioned previously, from Hall (1986), in order to have stationary disturbances we must choose $\alpha_{0}$ and $\beta_{0}$ so that the wall shear is zero at zeroth order. Then $\alpha_{0} \hat{u}_{0}+\beta_{0} \hat{v}_{0} / r \rightarrow 0$ as $z_{B} \rightarrow 0$. At the next order of $\varepsilon$ the solutions which match with the upper deck solutions have the same form as (4.9) but with amplitude $f$ replaced by $g$. Thus, we have $\hat{p}_{1}=g$.

We find that the first order terms proportional to $\delta^{2} E^{2}$ have the same forms as (4.9) but with $\alpha_{0}, \beta_{0}$ and $f$ replaced by $2 \alpha_{0}, 2 \beta_{0}$ and $f_{2}$, respectively. Similarly, the first order terms proportional to $\delta^{3} E$ which match with the solutions in the upper deck have the same form as (4.9) but with the amplitude $f$ replaced by $f_{4}$. In both cases the nonlinear effects have no contribution until the third order solutions.

We now require a lower deck to reduce the slip velocities to zero at the surface of the disc. The viscous terms in the governing equations will now play a part in the first order solutions at $0(\delta E), 0\left(\delta^{2} E^{2}\right)$ and $0\left(\delta^{3} E\right)$ and the nonlinear effects will arise at the solution of the next order terms in $\epsilon$ at $0\left(\delta^{2} E^{2}\right)$ and $0\left(\delta^{3} E\right)$. 


\subsection{The Lower-Deck Expansions}

We introduce the vertical coordinate $\xi$ where $z=\varepsilon^{9} \xi$ so that $\xi$ is $0(1)$ in the lower deck. For small values of $z_{B}$ we can expand the basic flow quantities $u_{B}, v_{B}, R_{0}$ Then, in terms of $\xi$

$$
\begin{aligned}
& u_{B}=\varepsilon \xi u_{B 1}+\varepsilon^{2} \xi^{2} u_{B 2}+\cdots, \quad v_{B}=\varepsilon \xi v_{B 1}+\varepsilon^{2} \xi^{2} v_{B 2}+\cdots, \\
& R_{0}=R_{0 W}+\varepsilon \xi R_{01}+\varepsilon^{2} \xi^{2} R_{02}+\cdots, \quad T_{B}=T_{B W}+\varepsilon \xi T_{B 1}+\varepsilon^{2} \xi^{2} T_{B 2}+\cdots,
\end{aligned}
$$

where $R_{0 W}=R_{0}\left(z_{B}=0\right)$. The other coefficients are defined by

$$
u_{B j}=\left.\frac{1}{j !} \frac{\partial^{j} u_{B}}{\partial z_{B}^{j}}\right|_{z_{B}=0},
$$

and similarly for $v_{B j}, R_{0 j}, T_{B j}, j=1,2, \ldots$. The coefficients will depend on $r$ and then the condition of zero wall shear can be written as $\alpha_{0} u_{B 1}+\beta_{0} v_{B 1} / r=0$. Hence, in order for the expansions of the disturbance in the lower deck to match with those in the main deck as $\xi \rightarrow \infty$, from (4.9) and (4.10) the radial velocity disturbance must have the form

$$
\begin{aligned}
U= & \delta\left(\frac{\Gamma f}{\varepsilon \beta_{0}^{2}}\left(u_{B 1}+2 \varepsilon \xi u_{B 2}+\cdots\right)+\frac{U_{0}}{\varepsilon}+U_{1}+\varepsilon U_{2}+\cdots\right) E \\
& +\delta^{2}\left(\left(\frac{\Gamma f_{2}}{\varepsilon^{4} 2 / B_{0}^{2}}\left(u_{B 1}+2 \varepsilon \xi u_{B 2}+\cdots\right)+\frac{U_{20}}{\varepsilon^{4}}+\frac{U_{21}}{\varepsilon^{3}}+\cdots\right) E^{2}+\frac{U_{m 0}}{\varepsilon^{3}}+\frac{U_{m 1}}{\varepsilon^{2}}+\cdots\right) \\
& +\delta^{3}\left(\left(\frac{U_{30}}{\varepsilon^{7}}+\frac{U_{31}}{\varepsilon^{6}}+\cdots\right) E^{3}+\left(\frac{\Gamma f_{4}}{\varepsilon^{7} \beta_{0}^{2}}\left(u_{B 1}+2 \varepsilon \xi u_{B 2}+\cdots\right)+\frac{U_{10}}{\varepsilon^{7}}+\frac{U_{11}}{\varepsilon^{6}}+\cdots\right) E\right) \\
& +0\left(\delta^{4}\right)+c . c .
\end{aligned}
$$

The azimuthal velocity, density and temperature perturbations have similar expressions to (4.11a) in the lower deck. The normal velocity perturbation has the form

$$
\begin{aligned}
W & =\delta\left(-\frac{i \Gamma f \varepsilon^{5}}{\beta_{0}^{2}}\left[\left(\alpha_{0} u_{B 2}+\frac{\beta_{0}}{r} v_{B 2}\right) \xi^{2}+\varepsilon\left(\alpha_{0} u_{B 3}+\frac{\beta_{0}}{r} v_{B 3}\right) \xi^{3}+\cdots\right]+\varepsilon^{6} W_{0}+\varepsilon^{7} W_{1}+\cdots\right) E \\
& +\delta^{2}\left(\left(\frac{-i \Gamma f_{2} \varepsilon^{2}}{\beta_{0}^{2}}\left[\left(\alpha_{0} u_{B 2}+\frac{\beta_{0}}{r} v_{B 2}\right) \xi^{2}+\cdots\right]+\varepsilon^{3} W_{20}+\varepsilon^{4} W_{21}+\cdots\right) E^{2}+\varepsilon^{4} W_{m 0}+\varepsilon^{5} W_{m 1}+\cdots\right) \\
& +\delta^{3}\left(\left(\frac{W_{30}}{\varepsilon}+W_{31}+\cdots\right) E^{3}+\left(\frac{-i \Gamma f_{4}}{\varepsilon \beta_{0}^{2}}\left[\left(\alpha_{0} u_{B 2}+\frac{\beta_{0}}{r} v_{B 2}\right) \xi^{2}+\cdots\right]\right.\right. \\
& \left.\left.+W_{10}+\varepsilon W_{11}+\cdots\right) E\right)+0\left(\delta^{4}\right)+c . c .
\end{aligned}
$$

while the pressure perturbation is given by 


$$
\begin{aligned}
P= & \delta\left(\varepsilon^{3} f+\varepsilon^{4} g+\cdots\right) E+\delta^{2}\left(\left(f_{2}+\varepsilon P_{21}+\cdots\right) E^{2}+\varepsilon^{10} P_{m 0}+\varepsilon^{11} P_{m 1}+\cdots\right) \\
& +\delta^{3}\left(\left(\frac{P_{30}}{\varepsilon^{3}}+\frac{P_{31}}{\varepsilon^{2}}+\cdots\right) E^{3}+\left(\frac{f_{4}}{\varepsilon^{3}}+\frac{P_{11}}{\varepsilon^{2}}+\cdots\right) E\right)+0\left(\delta^{4}\right)+c . c .
\end{aligned}
$$

Note that the mean flow terms in (4.11b) are larger than for the incompressible case. The perturbed flow given by (4.11) with the basic flow quantities given by (4.10) is substituted into the governing equations (2.2) - (2.5).

\subsection{The Linear Eigenrelation}

We equate coefficients of $\delta E$ in the governing equations for the perturbed flow in the lower deck. It is found that

$$
U_{0}=\frac{-\Gamma f u_{B 1}}{\beta_{0}^{2}} \frac{U\left(0, \sqrt{2} \Delta^{\frac{1}{4}} \xi\right)}{U(0,0)}
$$

where $\mathcal{U}$ is a parabolic cylinder functions (see, for example, Abramowitz and Stegun (1964)) and

$$
\Delta=\frac{i}{\mu_{B W} T_{B W}}\left(\alpha_{0} u_{B 2}+\frac{\beta_{0}}{r} v_{B 2}\right)=-i \frac{r \alpha_{0}}{2 T_{B W}^{2} \mu_{B W}^{2}},
$$

from the $r$ and $\theta$ momentum equations of the basic flow solution and using the condition (4.6a) on the effective wall shear. $V_{0}$ is determined from the following relation obtained from the continuity equation:

$$
\alpha_{0} U_{0}+\frac{\beta_{0}}{r} V_{0}=0
$$

From the energy equation we find that

$$
T_{10}=\frac{-\Gamma f T_{B 1}}{\beta_{0}^{2}} \frac{U\left(0, \sqrt{2} \sigma^{\frac{1}{4}} \Delta^{\frac{1}{4}} \xi\right)}{U(0,0)},
$$

and hence from the equation of state (2.3a)

$$
\rho_{10}=\frac{-\Gamma f R_{01}}{\beta_{0}^{2}} \frac{U\left(0, \sqrt{2} \sigma^{\frac{1}{4}} \Delta^{\frac{1}{4}} \xi\right)}{U(0,0)} .
$$

We note that for an adiabatic wall $T_{10}$ and $\rho_{10}$ will be equal to zero.

It is found that the functions $U_{1}$ and $V_{1}$ cannot be determined until the solution for $W_{0}$ is obtained. This is achieved by solving a third order ordinary differential equation similar to equation (4.16) in Hall (1986). Hence, $W_{0}$ is given by

$$
\begin{aligned}
W_{0}= & -i\left(\alpha_{1} u_{B 1}+\frac{\beta_{1}}{r} v_{B 1}\right) \frac{\Gamma f \xi}{\beta_{0}^{2}}+\Delta^{-\frac{3}{4}}\left[\left(\alpha_{0}^{2}+\frac{\beta_{0}^{2}}{r^{2}}\right) \frac{f F_{1}(s)}{\mu_{B W}}+2 i\left(\alpha_{0}^{2}+\frac{\beta_{0}^{2}}{r^{2}}\right) \frac{r \Gamma f u_{B 1} F_{2}(s)}{\beta_{0}^{3} T_{B W} U(0,0) T_{B W}}\right. \\
& \left.-\frac{3 i \alpha_{0} r \Gamma f R_{01} F_{3}(s)}{\beta_{0}^{2} \mu_{B W} U(0,0)}+\frac{i(1-\sigma) \Gamma f R_{01} r \alpha_{0} F_{4}(s)}{2 \beta_{0}^{2} \mu_{B W} U(0,0)}\right]+\frac{i \Gamma g r \alpha_{0} \xi^{2}}{2 \beta_{0}^{2} T_{B W} \mu_{B W}}
\end{aligned}
$$


where $s=\Delta^{\frac{1}{4}} \xi$. The function $F_{1}$ satisfies the equation

$$
F_{1}^{\prime \prime \prime}-s^{2} F_{1}^{\prime}+2 s F_{1}=1,
$$

while $F_{2}, F_{3}$ and $F_{4}$ satisfy the same equation as $F_{1}$ but with the right-hand-side replaced by $U(0, \sqrt{2} s), \frac{d}{d s}\left[s U\left(0, \sqrt{2} \sigma^{\frac{1}{4}} s\right)\right]$ and $s^{4} U\left(0, \sqrt{2} \sigma^{\frac{1}{4}} s\right)$, respectively. The boundary conditions are $F_{i}(0)=F_{i}(\infty)=0, i=1,2,3,4$. The continuity equation relating $U_{2}$ and $V_{2}$ is

$$
i\left(\alpha_{0} U_{2}+\frac{\beta_{0}}{r} V_{2}\right)+i\left(\alpha_{1} U_{0}+\frac{\beta_{1}}{r} V_{0}\right)+\frac{i \Gamma f}{\beta_{0}^{2}}\left(\alpha_{1} u_{B 1}+\frac{\beta_{1}}{r} v_{B 1}\right)+\frac{\partial W_{0}}{\partial \xi}-\frac{i \rho_{10} r \alpha_{0} \xi^{2}}{2 \mu_{B W}}=0 .
$$

Satisfying the boundary conditions at $\xi=0$ in (4.13) and using (4.12) gives the eigenrelation

$$
\begin{gathered}
\left(\alpha_{0}^{2}+\frac{\beta_{0}^{2}}{r^{2}}\right) f F_{1}^{\prime}(0)+2 i\left(\alpha_{0}^{2}+\frac{\beta_{0}^{2}}{r^{2}}\right) \frac{r \Gamma f u_{B 1} F_{2}^{\prime}(0)}{\beta_{0}^{3} T_{B W} U(0,0)}-\frac{3 i \alpha_{0} r \Gamma f R_{01} F_{3}^{\prime}(0)}{\beta_{0}^{2} U(0,0)} \\
+\frac{i(1-\sigma) \Gamma f R_{01} \alpha_{0} r F_{4}^{\prime}(0)}{2 \beta_{0}^{2} U(0,0)}=(1+i) \frac{\Gamma f\left(\alpha_{0} r\right)^{\frac{1}{2}}}{2 \beta_{0}^{2} T_{B W}}\left(\alpha_{1} u_{B 1}+\frac{\beta_{1}}{r} v_{B 1}\right) .
\end{gathered}
$$

Since the right-hand-side of (4.14) is proportional to $(1+i)$ and $\alpha_{0}$ and $\beta_{0}$ are real we have

$$
\begin{aligned}
\left(\alpha_{0}^{2}+\frac{\beta_{0}^{2}}{r^{2}}\right) F_{1}^{\prime}(0)-(1-\sigma) & \frac{\Gamma R_{01} \alpha_{0} r F_{4}^{\prime}(0)}{2 \beta_{0}^{2} U(0,0)}=2\left(\alpha_{0}^{2}+\frac{\beta_{0}^{2}}{r^{2}}\right) \frac{r u_{B 1} \Gamma F_{2}^{\prime}(0)}{\beta_{0}^{3} T_{B W} U(0,0)} \\
& -\frac{3 \alpha_{0} r \Gamma R_{01} F_{3}^{\prime}(0)}{\beta_{0}^{2} \mathcal{U}(0,0)} .
\end{aligned}
$$

The values of $F_{1}^{\prime}(0)$ and $2 F_{2}^{\prime}(0) / U(0,0)$ evaluated by Hall $(1986)$ are given by

$$
\begin{aligned}
F_{1}^{\prime}(0) & =0.5991, \\
\frac{2 F_{2}^{\prime}(0)}{U(0,0)} & =0.4570 .
\end{aligned}
$$

The values for $F_{3}^{\prime}(0)$ and $F_{4}^{\prime}(0)$ depend on $\sigma$ and can be obtained in the same way as Hall (1986). For $\sigma=0.72$ we obtain

$$
\begin{aligned}
& F_{3}^{\prime}(0)=0.0192, \\
& F_{4}^{\prime}(0)=1.6972 .
\end{aligned}
$$

We shall consider solutions of the eigenrelation (4.15) for the cases of an adiabatic wall and an isothermal wall separately.

\subsubsection{An Adiabatic Wall}

In this case $R_{01}=0$ so that the eigenrelation (4.15) becomes

$$
F_{1}^{\prime}(0)=\frac{2 r u_{B 1} F_{2}^{\prime}(0)}{T_{B W} \beta_{0}^{3} U(0,0)}\left(\alpha_{0}^{2}+\frac{\beta_{0}^{2}}{r^{2}}\left(1-M_{r}^{2}\right)\right)^{\frac{1}{2}}
$$


using (4.4). Note that if $M_{r}=0$ and $T_{B W}=1$ the eigenrelation (4.16) reduces to the eigenrelation obtained by Hall (1986) for incompressible flow. Now let

$$
\Gamma_{0}=\left(\alpha_{0}^{2}+\frac{\beta_{0}^{2}}{r^{2}}\right)^{\frac{1}{2}}
$$

From the solution of the basic flow described in Section 3, for an adiabatic wall the temperature at the wall is given by

$$
T_{B W}=1+(\gamma-1) \frac{M_{r}^{2} \phi_{2}(0)}{4},
$$

where $\phi_{2}(0)=1.7899$ for $\sigma=0.72$ and the value of $\gamma$ for air is 1.4. We also have from (3.6b) and (3.8) that

$$
\begin{aligned}
& u_{B 1}=\frac{r \bar{u}_{0}}{T_{B W} C^{1 / 2}}, \\
& v_{B 1}=\frac{r \bar{v}_{0}}{T_{B W} C^{1 / 2}},
\end{aligned}
$$

where $\bar{u}_{0}$ and $\bar{v}_{0}$ are given by (3.9e). The eigenrelation (4.16) may be rearranged using (4.6) and (4.17) to give

$$
\Gamma_{0} r^{\frac{1}{2}}=\left(\frac{2 \bar{u}_{0} F_{2}^{\prime}(0)}{C^{1 / 2} T_{B W}^{2} F_{1}^{\prime}(0) \mathcal{U}(0,0)}\right)^{\frac{1}{2}}\left(1+\frac{\bar{v}_{0}^{2}}{\bar{u}_{0}^{2}}\right)^{\frac{1}{2}}\left(1+\frac{\bar{v}_{0}^{2}}{\bar{u}_{0}^{2}}-M_{r}^{2}\right)^{\frac{1}{4}} .
$$

Hence, from (4.18) $\Gamma_{0} r^{\frac{1}{2}}$ is a function of $M_{r}$ only. From the results obtained by Hall (1986) $\Gamma_{0} r^{\frac{1}{2}}=1.224$ for an incompressible flow where $\Gamma_{0}$ can be regarded as the effective wavenumber of the perturbed flow.

If we now equate real parts in the eigenrelation (4.14) we have, for real values of $\alpha_{0}$ and $\beta_{0}$,

$$
\left(\alpha_{0}^{2}+\frac{\beta_{0}^{2}}{r^{2}}\right) F_{1}^{\prime}(0)=\frac{\Gamma\left(\alpha_{0} r\right)^{\frac{1}{2}}}{2 \beta_{0}^{2} T_{B W}}\left(\alpha_{1} u_{B 1}+\frac{\beta_{1}}{r} v_{B 1}\right),
$$

which can be rearranged using (4.17) to give

$$
\left(\frac{\alpha_{1}}{\beta_{0}}-\frac{\alpha_{0} \beta_{1}}{\beta_{0}^{2}}\right) r^{\frac{3}{4}}=2\left(\Gamma_{0} r^{\frac{1}{2}}\right)^{\frac{3}{2}} \frac{F_{1}^{\prime}(0) T_{B W}^{2} C^{1 / 2}}{\left|\bar{u}_{0} \bar{v}_{0}\right|^{\frac{1}{2}}}\left(1+\frac{\bar{v}_{0}^{2}}{\bar{u}_{0}^{2}}-M_{r}^{2}\right)^{-\frac{1}{2}}\left(1+\frac{\bar{v}_{0}^{2}}{\bar{u}_{0}^{2}}\right)^{\frac{1}{4}} .
$$

The value of $\left(\alpha_{1} / \beta_{0}-\alpha_{0} \beta_{1} / \beta_{0}^{2}\right) r^{\frac{5}{4}}$ was found to be 2.312 by Hall (1986) for incompressible flow. The expression (4.20) gives a correction to the angle between the radius vector and the normal to the stationary vortices. The results given by (4.18) and (4.20) are the compressible generalization of the incompressible results obtained by Hall (1986). A discussion of these results is given in Section 5 for $\bar{T}_{\infty}=491.6^{\circ} R$. 


\subsubsection{An Isothermal Wall}

If we manipulate (4.15) we obtain

$\Gamma_{0} r^{\frac{1}{2}}=\left(1+\frac{\bar{v}_{0}^{2}}{\bar{u}_{0}^{2}}-M_{r}^{2}\right)^{\frac{1}{4}}\left[(\sigma-1) \frac{R_{01} \bar{v}_{0} F_{4}^{\prime}(0)}{2 \bar{u}_{0} U(0,0)}+\frac{2 \bar{u}_{0} F_{2}^{\prime}(0)}{C^{1 / 2} T_{B W}^{2} U(0,0)}\left(1+\frac{\bar{v}_{0}^{2}}{\bar{u}_{0}^{2}}\right)+\frac{3 \bar{v}_{0} R_{01} F_{3}^{\prime}(0)}{\bar{u}_{0} U(0,0)}\right]^{\frac{1}{2}}\left[F_{1}^{\prime}(0)\right]^{-\frac{1}{2}}$.

From the solution for the basic flow

$$
T_{B 1}=\left[-\left(T_{B W}-1\right) A+(\gamma-1) \frac{M_{r}^{2}}{4} \phi_{2}^{\prime}(0)\right] /\left(C^{1 / 2} T_{B W}\right),
$$

where, for $\sigma=0.72, A=0.3286$ and $\phi_{2}^{\prime}(0)=0.9348$. From the equation of state $R_{01}=$ $-T_{B 1} / T_{B W}^{2}$. In order to evaluate (4.21) for values of $M_{r}$ the temperature of the wall must be specified. $T_{B W}$ is the non-dimensional temperature at the wall, defined by $T_{B W}=\bar{T}_{B W} / \bar{T}_{\infty}$. Hence, $T_{B W}$ is the ratio of the dimensional temperature at the wall to the free-stream dimensional temperature. Calculations are obtained for $\bar{T} \infty=491.6^{\circ} \mathrm{R}$ and for a range of values of $T_{B W}$. Figure 1 shows $\Gamma_{0} r^{\frac{1}{2}}$ as a function of $M_{r}$ for $\sigma=0.72$ for $T_{B W}=1.8,1.0,0.6$ and 0.2 , and also for the case of an adiabatic wall. We see that the effective wavenumber of the disturbed flow decreases as the local Mach number increases.

Equating real parts of (4.14) gives the same expression as for the adiabatic wall, i.e., (4.20), but of course the values of $\alpha_{0}$ and $\beta_{0}$ will be different, $\Gamma_{0}$ being given by (4.21). Figure 2 shows $\left(\alpha_{1} / \beta_{0}-\alpha_{0} \beta_{1} / \beta_{0}^{2}\right) r^{\frac{s}{4}}$ as a function of $M_{\mathrm{r}}$ for $\sigma=0.72$ for $T_{B W}=1.8,1.0,0.6$, and 0.2 , as well as for an adiabatic wall. We describe the orientation of the stationary vortices are given by the angle $\theta_{0}$, where $\theta_{0}$ is the angle between the tangent to the vortices and the normal to the radius vector, then

$$
\tan \left(\frac{\pi}{2}-\theta_{0}\right)=\frac{\alpha r}{\beta}=\frac{\alpha_{0} r}{\beta_{0}}+\varepsilon^{2} r^{-\frac{1}{4}}\left(\frac{\alpha_{1}}{\beta_{0}}-\frac{\alpha_{0} \beta_{1}}{\beta_{0}^{2}}\right) r^{\frac{5}{4}}+\cdots
$$

Thus, from Figure 2 we see that $\theta_{0}$ decreases as the local Mach number increases for $T_{B W} \geq 0.6$ but for highly cooled walls with $T_{B W}<0.6 \quad \theta_{0}$ increases as the local Mach number increases. The significance of these results is discussed in Section 5.

\subsection{The Nonlinear Solution}

We now consider the terms arising from the nonlinear interactions in the governing equations in the lower deck. We substitute the expansions (4.11) for the perturbed flow into the governing equations $(2.2)-(2.5)$ and equate coefficients of $\delta^{2} E^{2}$. We find the solutions

$$
U_{20}=-\frac{\Gamma f_{2} u_{B 1}}{2 \beta_{0}^{2}} \frac{U\left(0, \sqrt{2}(2 \Delta)^{\frac{1}{4}} \xi\right)}{U(0,0)}
$$




$$
T_{20}=-\frac{\Gamma f_{2} T_{B 1}}{2 \beta_{0}^{2}} \frac{U\left(0, \sqrt{2} \sigma^{\frac{1}{4}}(2 \Delta)^{\frac{1}{4}} \xi\right)}{U(0,0)}
$$

where $V_{20}$ and $\rho_{20}$ are determined from

$$
\begin{aligned}
& \alpha_{0} U_{20}+\frac{\beta_{0}}{r} V_{20}=0 \\
& R_{01} T_{20}-T_{B 1} \rho_{20}=0,
\end{aligned}
$$

respectively. In the same way as for the linear terms, $U_{21}$ and $V_{21}$ cannot be determined until $W_{20}$ is obtained. The solution for $W_{20}$ is the same as that obtained for $W_{0},(4.12 \mathrm{~d})$, with $\alpha_{j}, \beta_{j}, f$ replaced by $2 \alpha_{j}, 2 \beta_{j}, f_{2}$, respectively, for $j=0,1,2$. Satisfying the boundary conditions at the wall in the continuity equation gives the following expression relating $f_{2}$ to $f$ :

$$
\begin{gathered}
f_{2}\left[4\left(\alpha_{0}^{2}+\frac{\beta_{0}^{2}}{r^{2}}\right) \frac{F_{1}^{\prime}(0)}{\mu_{B W}}+2 i\left(\alpha_{0}^{2}+\frac{\beta_{0}^{2}}{r^{2}}\right) \frac{\Gamma r u_{B 1} F_{2}^{\prime}(0)}{\beta_{0}^{3} T_{B W} U(0,0) \mu_{B W}}-\frac{3 i \alpha_{0} r \Gamma R_{01} F_{3}^{\prime}(0)}{\beta_{0}^{2} \mu_{B W} U(0,0)}\right. \\
\left.+\frac{i(1-\sigma) \alpha_{0} r \Gamma R_{01} F_{4}^{\prime}(0)}{2 \beta_{0}^{2} \mu_{B W} U(0,0)}-i(2 \Delta)^{\frac{1}{2}} \frac{\Gamma}{\beta_{0}^{2}}\left(\alpha_{1} u_{B_{1}}+\frac{\beta_{1}}{r} v_{B 1}\right)\right]=2 i(2 \Delta)^{\frac{1}{2}} \frac{\Gamma^{2} f^{2}}{\beta_{0}^{4}}\left(\alpha_{0} u_{B 2}+\frac{\beta_{0}}{r} v_{B 2}\right) .
\end{gathered}
$$

Note that $f_{2}$ is proportional to $f^{2} . F_{3}^{\prime}(0)$ and $F_{4}^{\prime}(0)$ may be eliminated from (4.22) by using the linear eigenrelation (4.15).

Now if the same procedure is followed for the solution of the terms proportional to $\delta^{3} E$ in the perturbation quantities solutions can be obtained for $U_{10}, V_{10}, W_{10}, T_{10}$ and $\rho_{10}$. These are found to be identical to the linear solutions (4.12) with $f$ replaced by the amplitude $f_{4}$. However, the resulting eigenrelation here is

$$
\begin{gathered}
\frac{\Delta^{-1 / 2} f_{4}}{\mu_{B W}}\left\{-\frac{(1+i) \Gamma\left(\alpha_{0} r\right)^{1 / 2}}{2 \beta_{0}^{2} T_{B W}}\left(\alpha_{1} u_{B 1}+\frac{\beta_{1}}{r} v_{B 1}\right)+\left(\alpha_{0}^{2}+\frac{\beta_{0}^{2}}{r^{2}}\right) F_{1}^{\prime}(0)+2 i\left(\alpha_{0}^{2}+\frac{\beta_{0}^{2}}{r^{2}}\right) \frac{\Gamma u_{B 1} r F_{2}^{\prime}(0)}{\beta_{0}^{3} T_{B W} U(0,0)}\right. \\
\left.-\frac{3 i \Gamma R_{01} \alpha_{0} r F_{3}^{\prime}(0)}{\beta_{0}^{2} U(0,0)}+\frac{i(1-\sigma) \Gamma R_{01} \alpha_{0} r F_{4}^{\prime}(0)}{2 \beta_{0}^{2} U(0,0)}\right\}=\frac{i \Gamma^{2} f^{*} f_{2}}{\beta_{0}^{4}}\left(\alpha_{0} u_{B 2}+\frac{\beta_{0}}{r} v_{B 2}\right),
\end{gathered}
$$

where $*$ denotes complex conjugate. Since, from (4.22) $f_{2}$ is proportional to $f^{2}$ and from the linear eigenrelation (4.14) the left-hand-side of (4.23) is zero, this implies that $f \equiv 0$. In order to determine whether the nonlinear effects are stabilizing or destabilizing we must move a sufficient distance from the position of neutral stability.

We wish to determine an amplitude equation for the disturbance amplitude. This will involve derivatives with respect to $r$ and nonlinear terms. In order to obtain the required equation, from the results of MacKerrell (1987) we find it is necessary to perturb the solution a distance of $O(\varepsilon)$ from the neutral position. We must also choose $\delta=\varepsilon^{\frac{7}{2}}$ so that 
the appropriate nonlinear terms will arise in the eigenrelation. Thus, we define a new variable $r_{1}$ by

$$
r=\bar{r}+\varepsilon r_{1},
$$

where $\bar{r}$ is the position of neutral stability. Hence, we can write

$$
\frac{\partial}{\partial r}=\frac{i \alpha_{0}}{\varepsilon^{4}}+\frac{i \alpha_{1}}{\varepsilon^{2}}+\frac{1}{\varepsilon} \frac{\partial}{\partial r_{1}}+\cdots
$$

for the terms proportional to $E$ and similarly for the $0\left(E^{2}\right)$ and $0\left(E^{3}\right)$ terms. Thus, $\alpha_{2}$ may be effectively replaced by $-i \partial / \partial r_{1}$ in the equations previously obtained. The required eigenrelation will involve terms proportional to $f, r_{1} f, d f / d r_{1}$ and $f|f|^{2}$.

We replace $r$ from (4.24) in the governing equations and rewrite the expansions of the perturbation quantities in the lower deck. For example,

$$
\begin{aligned}
U= & \varepsilon^{\frac{\tau}{2}}\left\{\frac{\Gamma f}{\varepsilon \beta_{0}^{2}}\left(u_{B 1}+2 \varepsilon \xi u_{B 2}+\cdots\right)+\frac{\tilde{U}_{0}}{\varepsilon}+\tilde{U}_{1}+\cdots E\right. \\
& +\varepsilon^{\frac{1}{2}}\left(\frac{\Gamma f_{2}}{2 \varepsilon \beta_{0}^{2}}\left(u_{B 1}+2 \varepsilon \xi u_{B 2}+\cdots\right)+\frac{\tilde{U}_{20}}{\varepsilon}+\cdots\right) E^{2}+\varepsilon^{\frac{1}{2}}\left(\tilde{U}_{m 0}+\varepsilon \tilde{U}_{m 1}+\cdots\right) \\
& \left.+\varepsilon\left(\frac{\Gamma f_{3}}{3 \varepsilon \beta_{0}^{2}}\left(u_{B 1}+2 \varepsilon \xi u_{B 2}+\cdots\right)+\frac{\tilde{U}_{30}}{\varepsilon}+\cdots\right) E^{3}\right\}+0\left(\varepsilon^{4}\right)+c . c . \\
W= & \varepsilon^{\frac{7}{2}}\left\{\left(-\frac{i \Gamma f \varepsilon^{5}}{\beta_{0}^{2}}\left[\left(\alpha_{0} u_{B 2}+\frac{\beta_{0}}{r} v_{B 2}\right) \xi^{2}+\cdots\right]+\varepsilon^{6} \tilde{W}_{0}+\varepsilon^{7} \tilde{W}_{1}+\cdots\right) E\right. \\
& +\varepsilon^{\frac{1}{2}}\left(-\frac{i \Gamma f_{2} \varepsilon^{5}}{\beta_{0}^{2}}\left[\left(\alpha_{0} u_{B 2}+\frac{\beta_{0}}{r} v_{B 2}\right) \xi^{2}+\cdots\right]+\varepsilon^{6} \tilde{W}_{20}+\cdots\right) E^{2}+\varepsilon^{\frac{15}{2}}\left(\tilde{W}_{m 0}+\varepsilon \hat{W}_{m 1}+\cdots\right) \\
& \left.+\varepsilon\left(-\frac{i \Gamma f_{3} \varepsilon^{5}}{\beta_{0}^{2}}\left[\left(\alpha_{0} u_{B 2}+\frac{\beta_{0}}{r} v_{B 2}\right) \xi^{2}+\cdots\right]+\varepsilon^{6} \tilde{W}_{30}+\cdots\right) E^{3}\right\}+0\left(\varepsilon^{10}\right)+c . c .
\end{aligned}
$$

In order to obtain the required eigenrelation we need to determine $\tilde{W}_{1}$. The linear terms may be obtained directly from the equation for $W_{1}$. The terms occurring from expanding $r$ are obtained from the $0(\varepsilon)$ terms obtained by expanding $r$ from (4.24) in the equation for $W_{0}$. The nonlinear terms will simply be the nonlinear terms occurring in the equation for $W_{10}$. Then satisfying the boundary conditions at $\xi=0$ gives the solvability condition

$$
\frac{\partial \tilde{W}_{1}}{\partial \xi}=0 \text { at } \xi=0
$$


This condition leads to the following relation:

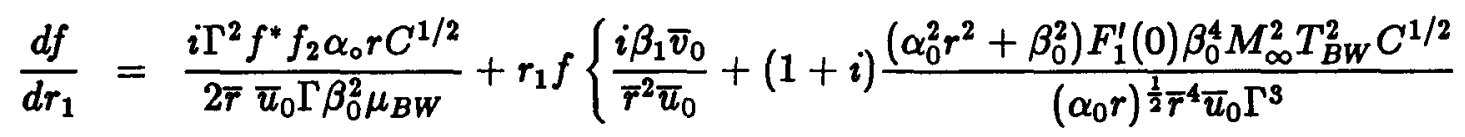

$$
\begin{aligned}
& -(1+3 i) \frac{\beta_{0}^{2}\left(\alpha_{0}^{2} r^{2}+\beta_{0}^{2}\right) F_{1}^{\prime}(0) T_{B W}^{2} C^{1 / 2}}{\left(\alpha_{0} r\right)^{\frac{1}{2} \bar{r}^{4}} \bar{u}_{0} \Gamma}+(1-i) \frac{\left(\alpha_{0}^{2} r^{2}+\beta_{0}^{2}\right) F_{1}^{\prime}(0) \beta_{0}^{2} 2 b T_{B W}^{2} C^{1 / 2}}{\left(\alpha_{0} r\right)^{\frac{1}{2}} \bar{r}^{2} \bar{u}_{0} \Gamma R_{01}} \\
& \left.-(1-i) \frac{4\left(\alpha_{0}^{2} r^{2}+\beta_{0}^{2}\right) F_{2}^{\prime}(0) b}{\left(\alpha_{0} r\right)^{\frac{1}{2}} \beta_{0} R_{01} U(0,0)}\right\} \\
& +\frac{(1+i) \beta_{0}^{2} T_{B W}^{2} \mu_{B W} C^{1 / 2}}{\left(\alpha_{0} r\right)^{\frac{1}{2}} \bar{r} \bar{u}_{0} \Gamma} \int_{0}^{\infty} \frac{s u(0, \sqrt{2} s)}{U(0,0)} R H S\left(\xi=\Delta^{-\frac{1}{4}} s\right) d s .
\end{aligned}
$$

Here $b=(\gamma-1) M_{r}^{2} \phi_{2}^{\prime}(0) /\left(4 T_{B W}^{3} C^{1 / 2}\right)$ and is equal to zero for an adiabatic wall. The terms included in the expression RHS are linear terms depending on the functions $\tilde{\rho}_{L 0}, \tilde{\rho}_{L 1}, \tilde{W}_{0}, \tilde{U}_{0}, \tilde{U}_{1}, f$ and $g$ and so can be written as

$$
R H S(\xi)=c_{1}(\xi) f(r)
$$

We now follow the incompressible work of MacKerrell (1987) and write the eigenrelation (4.25) in the form

$$
\frac{d f}{d r_{1}}=\left(C_{1 r}+i C_{1 i}\right) f|f|^{2}+r_{1} f\left(C_{2 r}+i C_{2 i}\right)+f\left(C_{3 r}+i C_{3 i}\right)
$$

where $C_{k r}$ and $C_{k i}$ are constants for $k=1,2,3$. Now multiply (4.26) by $f^{*}$ and add the complex conjugate to give

$$
\frac{d}{d r_{1}}\left(|f|^{2}\right)=2 C_{1 r}|f|^{4}+2 C_{2 r}\left(r_{1}+\frac{C_{3 r}}{C_{2 r}}\right)|f|^{2}
$$

In order to simplify (4.27) for $C_{2 r} \neq 0$ we introduce a new variable $r_{1}^{\prime}=r_{1}+C_{3 r} / C_{2 r}$. Then (4.27) becomes

$$
\frac{d}{d r_{1}^{\prime}}\left(|f|^{2}\right)=2 C_{1 r}|f|^{4}+2 C_{2 r} r_{1}^{\prime}|f|^{2} .
$$

From (4.25) and the expression for $f_{2},(4.22)$, after some manipulation we obtain

$$
C_{1 r}=\bar{r}^{-\frac{1 s}{4}} \frac{3 F_{1}^{\prime}(0)\left(\bar{r}^{\frac{1}{2}} \Gamma_{0}\right)^{\frac{3}{2}}}{2 \sqrt{2} \bar{u}_{0} T_{B W}^{2}|\lambda|^{2} \mu_{B W}^{4}}\left(1+\frac{\bar{v}_{0}^{2}}{\bar{u}_{0}^{2}}-M_{r}^{2}\right)^{\frac{3}{2}}\left|\frac{\bar{v}_{0}}{\bar{u}_{0}}\right|^{\frac{5}{2}}\left(1+\frac{\bar{v}_{0}^{2}}{\bar{u}_{0}^{2}}\right)^{\frac{1}{4}} C^{1 / 2}
$$

where

$$
|\lambda|^{2}=\frac{17\left(\bar{r}^{\frac{1}{2}} \Gamma_{0}\right)^{4}\left(F_{1}^{\prime}(0)\right)^{2}}{\mu_{B W}^{2}}+\frac{\left|\bar{u}_{0} \bar{v}_{0}\right| \bar{r}^{\frac{1}{2}} \Gamma_{0}}{\mu_{B W}^{2} T_{B W}^{4} C}\left(1+\frac{\bar{v}_{0}^{2}}{\bar{u}_{0}^{2}}\right)^{-\frac{1}{2}}\left(1+\frac{\bar{v}_{0}^{2}}{\bar{u}_{0}^{2}}-M_{r}^{2}\right)\left[\left(\frac{\alpha_{1}}{\beta_{0}}-\frac{\beta_{1} \alpha_{0}}{\beta_{0}^{2}}\right) r^{-\frac{s}{4}}\right]^{2}
$$




$$
-\frac{10\left(\bar{r}^{\frac{1}{2}} \Gamma_{0}\right)^{\frac{5}{2}} F_{1}^{\prime}(0)\left|\bar{u}_{0} \bar{v}_{0}\right|^{\frac{1}{2}}}{\sqrt{2} T_{B W}^{2} \mu_{B W}^{2} C^{1 / 2}}\left(1+\frac{\bar{v}_{0}^{2}}{\bar{u}_{0}^{2}}\right)^{-\frac{1}{4}}\left(1+\frac{\bar{v}_{0}^{2}}{\bar{u}_{0}^{2}}-M_{r}^{2}\right)^{\frac{1}{2}}\left(\frac{\alpha_{1}}{\beta_{0}}-\frac{\beta_{1} \alpha_{0}}{\beta_{0}^{2}}\right) \bar{r}^{\frac{5}{4}} .
$$

Figure 3 shows $\bar{r}^{13 / 4} C_{1 r}$ as a function $M_{r}$ for an adiabatic wall and for an isothermal wall with $T_{B W}=1.8,1.0,0.6,0.2$. We find that $C_{1 r}$ is always positive and thus the nonlinear effects are destabilizing. We find that $\bar{r}^{13 / 4} C_{1 r}$ is less than the incompressible value of $\bar{r}^{13 / 4} C_{1 r}=2.357$ obtained by Mackerell (1987) for both cases of an adiabatic wall and an isothermal wall with $T_{B W} \geq 1$.0. However, for an isothermal wall with $T_{B W}<1.0 \quad \bar{r}^{13 / 4} C_{1 r}$ is greater than the incompressible value at $M_{r}=0.05$. Note that $C_{1 r}$ decreases as the local Mach number increases for all values of $T_{B W}$. Thus, for a highly cooled wall the destabilizing effects of nonlinearity are greater for a compressible flow than for an incompressible flow. From (4.25)

$$
\begin{aligned}
C_{2 r}= & -\bar{r}^{-\frac{7}{4}} \frac{\left(\bar{r}^{\frac{1}{2}} \Gamma_{0}\right)^{\frac{5}{2}}}{\left|\bar{u}_{0} \bar{v}_{0}\right|^{\frac{1}{2}}} F_{1}^{\prime}(0) T_{B W}^{2}\left(1+\frac{\bar{v}_{0}^{2}}{\bar{u}_{0}^{2}}\right)^{\frac{s}{4}}\left(1+\frac{\bar{v}_{0}^{2}}{\bar{u}_{0}^{2}}-M_{r}^{2}\right)^{-\frac{3}{2}} C^{1 / 2} \\
& +\bar{r}^{-\frac{7}{4}} \frac{\left(\bar{r}^{\frac{1}{2}} \Gamma_{0}\right)^{\frac{1}{2}}(\gamma-1) M_{r}^{2} \phi_{2}^{\prime}(0) \bar{u}_{0}^{\frac{1}{2}}}{\left|\bar{v}_{0}\right|^{\frac{1}{2}}\left[\left(T_{B W}-1\right) A-(\gamma-1) M_{r}^{2} \phi_{2}^{\prime}(0) / 4\right]}\left(1+\frac{\bar{v}_{0}^{2}}{\bar{u}_{0}^{2}}\right)^{\frac{3}{4}} \\
& \times\left[\frac{F_{1}^{\prime}(0)\left(\bar{r}^{\frac{1}{2}} \Gamma_{0}\right)^{2} T_{B W}^{2} C^{1 / 2}}{2 \bar{u}_{0}}\left(1+\frac{\bar{v}_{0}^{2}}{\bar{u}_{0}^{2}}\right)^{-1}\left(1+\frac{\bar{v}_{0}^{2}}{\bar{u}_{0}^{2}}-M_{r}^{2}\right)^{-\frac{1}{2}}-\frac{F_{2}^{\prime}(0)}{U(0,0)}\right] .
\end{aligned}
$$

In Figure $4 \vec{r}^{7 / 4} C_{2 r}$ is plotted as a function of $M_{r}$ for an adiabatic wall and for an isothermal wall with $T_{B W}=1.8,1.0,0.6,0.2,0.15$. We see that for the range of local Mach number where the solutions are valid and for $T_{B W}>0.15$ that $C_{2 r}$ is negative. Thus, in this case the amplitude of the disturbance increases or decreases depending on whether $r$ is less than or greater than the neutral value. However, for $T_{B W} \leq 0.15$ we find that $C_{2 r}$ becomes positive as $M_{r}$ increases. Thus, in this situation the amplitude of the disturbance increases as the distance from the neutral radius is increased. For an adiabatic wall and for an isothermal wall with $T_{B W} \geq 0.6$ we find that $C_{2 r}$ decreases as the local Mach number increases. However, for an isothermal wall with $T_{B W}<0.6$ there will be a range of the local Mach number where $C_{2 r}$ increases as the local Mach number increases. We note that the incompressible value of $\bar{r}^{7 / 4} C_{2 r}$ found by MacKerrell (1987) is equal to -0.903 and we see that for an adiabatic wall and an isothermal wall with $T_{B W} \geq 1$ that here $\bar{r}^{7 / 4} C_{2 r}$ is less than the incompressible value.

With the substitutions

$$
|f|^{2}=y\left|C_{2 r}\right|^{1 / 2} /\left(2 C_{1 r}\right) \text { and } r_{1}^{\prime}=x /\left|C_{2 r}^{1 / 2}\right|,
$$

for $C_{2 r} \neq 0,(4.28)$ becomes

$$
\frac{d y}{d x}= \pm 2 x y+y^{2}
$$


with the positive sign taken if $C_{2 r}>0$ and the negative sign taken if $C_{2 r}<0$. If $C_{2 r}=0$ use the substitutions

$$
|f|^{2}=y\left(2 C_{3 r}\right) /\left(2 C_{1 r}\right) \text { and } r_{1}=x /\left(2 C_{3 r}\right)
$$

so that (4.27) becomes

$$
\frac{d y}{d x}=y^{2}+y
$$

The solutions of (4.29) and (4.30) are

$$
\begin{gathered}
y(x)=e^{x^{2}} /\left(1 / y_{0}-\int_{0}^{x} e^{t^{2}} d t\right), \quad C_{2 r}>0, \\
y(x)=y_{0} e^{x} /\left(1+y_{0}\left(1-e^{x}\right)\right), \quad C_{2 r}=0, \\
y(x)=e^{-x^{2}} /\left(1 / y_{0}-(\sqrt{\pi} \operatorname{erf} x) / 2\right), \quad C_{2 r}<0,
\end{gathered}
$$

where

$$
y_{0}=\left|f\left(r_{1}^{\prime}=0\right)\right|^{2} \frac{2 C_{1 r}}{\left|C_{2 r}\right|^{1 / 2}}, \quad C_{2 r} \neq 0
$$

and

$$
y_{0}=\left|f\left(r_{1}^{\prime}=0\right)\right|^{2} 2 C_{1 r} / 2\left(C_{3 r}\right), \quad C_{2 r}=0 .
$$

The result (4.31c) is the generalization of the result obtained by MacKerrell (1987) where now $C_{1 r}$ and $C_{2 r}$ depend on $T_{B W}$ and $M_{r}$. Hence, for $C_{2 r}<0$ the same results hold for the compressible problem as for the incompressible problem. Thus, there exists a critical value of the initial amplitude of the disturbance. For values below this critical value $\left(y_{0}=2 / \sqrt{\pi}\right)$ the disturbance will grow initially but will eventually decay to zero. Thus, we will have a stable solution. For initial amplitudes above this critical value the disturbance becomes exponentially large at a particular radius and does not decay. Thus, in this case the solution will be unstable. For $C_{2 r} \geq 0$ we have growing solutions terminating at finite values of the radius. Thus there are no stable solutions for $C_{2 r} \geq 0$. Figure 5 shows $y$ as a function of $x$ for different values of $y_{0}$ from (4.31c) for $C_{2 r}<0$.

From the results of MacKerrell (1987) for an incompressible flow $\bar{r}^{-19 / 8}\left(-C_{2 r}\right)^{1 / 2} /\left(2 C_{1 r}\right)=$ 0.202. Figure 6 shows $\bar{r}^{-19 / 8}|f(0)|^{2} / y_{0}=\bar{r}^{-19 / 8}\left(-C_{2 r}\right)^{1 / 2} /\left(2 C_{1 r}\right)$, for $C_{2 r}<0$, as function of $M_{r}$ for an adiabatic wall and for an isothermal wall with $T_{B W}=1.8,1.0,0.6,0.2$. Thus, we see that $\bar{r}^{-19 / 8}\left(-C_{2 r}\right)^{1 / 2} /\left(2 C_{1 r}\right)$ is greater than the incompressible value for an adiabatic wall and for an isothermal wall with $T_{B W} \geq 1.0$ but less than this value for a range of the local Mach number when $T_{B W}<1.0$.

Thus, for an adiabatic wall and an isothermal wall with $T_{B W} \geq 1.0$ we require a larger value of the initial amplitude of the disturbance in the compressible case than in the incompressible case for the solution to become unstable. However, for some values 
of the local Mach number for an isothermal wall with $T_{B W}<1.0$ we require a smaller value of the initial amplitude of the disturbance in the compressible case than in the incompressible case for the solution to become unstable. Clearly, from Figure 6, as the local Mach number increases the threshold amplitude is increased and thus we require a greater initial amplitude for the disturbed flow to become unstable for larger values of the local Mach number.

\section{CONCLUSION}

The main conclusion to be drawn is that the effects of compressibility greatly alter these stationary viscous modes of instability since they cannot exist for large enough Mach number. We find that the results for a compressible flow, when these stationary modes exist, are similar to those obtained by MacKerrell (1987) for an incompressible flow. The main difference occurs for highly cooled walls. For instance, the finite amplitude of the disturbance solution increases or decreases depending on whether $r$ is less than or greater than the neutral position. In the same way as for the incompressible problem for particular values of $M_{r}$ and $T_{B W}$ we have the idea of a threshold solution as described by Stuart (1971) for the finite disturbance amplitude. Thus, there exists a critical value of the amplitude of the initial disturbance below which the disturbance eventually decays to zero as the distance from the position of neutral stability is increased and the solution will be stable. However, if the initial amplitude of the disturbance is above the critical value the solution will end in a singularity at a finite radius and the solution will be unstable. For highly cooled walls $\left(T_{B W} \leq 0.15\right)$ this threshold solution does not exist thus we have only unstable modes of instability. The nonlinear effects are found to be destabilizing for a compressible flow, which is also the case for an incompressible flow (see MacKerrell (1987)). It is found that for an adiabatic wall and an isothermal wall with $T_{B W} \geq 1.0$ the effects of nonlinearity are not as important here as for an incompressible flow and decrease as the local Mach number increases. But for an isothermal wall with $T_{B W}<1.0$ the effects of nonlinearity can be more important for a compressible flow than for an incompressible flow.

The weakly nonlinear analysis has enabled the effect of Mach number on the finite amplitude growth of a disturbance close to the neutral location to be described. Two cases of an adiabatic wall and an isothermal wall were investigated. It was found that the nature of the results was the same for an adiabatic wall and an isothermal wall with $T_{B W} \geq 1.0$. For these cases it was determined that the wavelength of the disturbance increases as the local Mach number increases. In addition, as the temperature of the wall is raised for an isothermal wall the disturbance wavelength increases. The orientation of the stationary vortices is described by the angle $\theta_{0}$ where $\theta_{0}$ is the angle between the tangent to the 
vortices and the normal to the radius vector. As the local Mach number is increased we find that $\theta_{0}$ decreases. For an isothermal wall as the temperature of the wall is increased $\theta_{0}$ decreases. Thus, we observe that for an isothermal wall with $T_{B W} \geq 1.0$ increasing either the local Mach number or the temperature of the wall produces the same effect. For an isothermal wall with $T_{B W}<1.0$ the same results apply with the exception that $\theta_{0}$ may increase as the local Mach number increases.

We now describe the main differences between our results for a compressible flow and those obtained by Hall (1986) and MacKerrell (1987) for an incompressible flow. We find that there is a larger mean flow correction in the normal direction in the lower deck for a compressible flow than for an incompressible flow, but otherwise the disturbance flow structures are the same. In Figure 1 we see that $\Gamma_{0} r^{1 / 2}$ is less than the value obtained by Hall (1986) for an adiabatic wall and also for an isothermal wall with $T_{B W} \geq 1.0$. Thus, the wavelength of the disturbance is longer in these situations than for an incompressible flow. However, for an isothermal wall with $T_{B W}<1.0$ the wavelength of the disturbance may be shorter than that for an incompressible flow. Figure 2 shows that $\left(\alpha_{1} / \beta_{0}-\alpha_{0} \beta_{1} / \beta_{0}^{2}\right) r^{5 / 4}$ is greater than the incompressible value obtained by Hall (1986) for an adiabatic wall and an isothermal wall with $T_{B W} \geq 1.0$. Hence, the angle of orientation of the stationary vortices $\theta_{0}$ is smaller for these cases than for an incompressible flow. For an isothermal with $T_{B W}<1.0 \theta_{0}$ may be larger than for an incompressible flow.

From the nonlinear analysis we found that $\bar{r}^{13 / 4} C_{1 r}$ is less than the value obtained by MacKerrell (1987) for an incompressible flow for an adiabatic wall and for an isothermal wall with $T_{B W} \geq 1.0$. Thus, in these cases the nonlinear effects are less important for a compressible flow than for an incompressible one although they are still destablishing. However, for an isothermal wall with $T_{B W}<1.0 \quad \bar{r}^{13 / 4} C_{1 r}$ may be greater than the incompressible value in which case the nonlinear effects will be stronger than for an incompressible flow. From Figure 4 we find that for highly cooled flows $C_{2 r}$ may be zero or positive. Thus, in this case there will be no threshold solution with only unstable modes of instability present. From Figure 6 we see that for $C_{2 r}<0 \quad \bar{r}^{-19 / 8}\left|f\left(r_{1}^{\prime}=0\right)\right|^{2} / y_{0}$ is greater than the value obtained by MacKerrell (1987) for an adiabatic wall and for an isothermal wall with $T_{B W}<1.0$. The solution (4.31c) breaks down when $y_{0}=2 / \sqrt{\pi}$ which is the same value as for an incompressible flow. Hence, for an adiabatic wall and an isothermal wall with $T_{B w} \geq 1.0$ for an unstable solution the initial amplitude of the disturbance for a compressible flow must be larger than that required for an incompressible flow. But for an isothermal wall with $T_{B w}<1.0$ we require a smaller initial amplitude for the solution to become unstable. In addition, for a highly cooled wall there is the possibility of no threshold solution, the solution being unstable in this case. Thus, an unstable mode of instability 
is more likely to occur for a compressible flow with a highly cooled wall than for an incompressible flow. For an adiabatic wall or an isothermal wall with $T_{B W} \geq 1.0$ the system must be forced even more strongly for a compressible flow than for an incompressible flow for these stationary modes of instability to be evident.

We found that these stationary viscous modes of instability do not occur above a critical supersonic local Mach number of about 1.57. This suggests that for small amplitude disturbances for values of the local Mach number greater than 1.57 the inviscid stationary modes described by Gregory, Stuart and Walker (1955) will be more dominant. Hence, for small amplitude disturbances it is unlikely that this mode would be observed in flows over bodies moving at very high Mach numbers. On the other hand, for values of the local Mach number less than $\mathbf{1 . 5 7}$, for highly cooled walls there is a strong possibility that the nonlinear effects may cause the early breakdown of the laminar flow.

\section{ACKNOWLEDGMENT}

I thank Professor P. Hall for suggesting this problem and for his useful comments on the first draft of this paper. My thanks also go to Dr. G. Erlebacher for discussions on this problem. 


\section{References}

[1] Abramowitz, M. and Stegun, I. A., 1964, Handbook of Mathematical Functions, Frankfurt: National Bureau of Standards.

[2] Cebeci, T. and Stewartson, K., 1980, "On stability and transition in three-dimensional flows," AIAA J. 18, 398-405.

[3] Faller, A. J. and Kaylor, R. E., 1966, "A numerical study of the instability of the laminar Ekman boundary layer," J. Atmos. Sci. 23, 466-480.

[4] Fedorov, B. I., Plavnik, G. Z., Prokhorov, I. V., and Zhukhovitskii, L. G., 1976, "Transitional flow conditions on a rotating disc," J. Engineering Physics 31, 14481453.

[5] Gregory, N., Stuart, J. T., and Walker, W. S., 1955, "On the stability of threedimensional boundary layers with application to the flow due to a rotating disc," Phil. Trans. Roy. Soc. A248, 155-199.

[6] Hall, P., 1986, "An asymptotic investigation of the stationary modes of instability of the boundary layer on a rotating disc," Proc. Roy. Soc. A406, 93-106.

[7] Kármán, Th. V., 1921, "Uber laminare und turbulente Reibung," Zeitschnift für angewante Mathematik und Mechanik 1, 233-252.

[8] Lilly, D. K., 1966, "On the instability of Ekman boundary flow," J. Atmos. Sci. 23, 481-494.

[9] Mack, L. M., 1985, "The wave pattern produced by point source on a rotating disc," AIAA Paper No. 85-0490.

[10] MacKerrell, S. O., 1987, "A nonlinear, asymptotic investigation of the stationary modes of instability of the three-dimensional boundary layer on a rotating disc," Proc. Roy. Soc. Lond. A413, 497-513.

[11] Malik, M. R., 1986, "The neutral curve for stationary disturbances in rotating disc flow," J. Fluid Mech. 164, 275-287.

[12] Malik, M. R., Wilkinson, S. P., and Orszag, S. A., 1981, "Instability and transition in rotating disc flow," AIAA J. 19, 1131-1138. 
[13] Riley, N., 1964, "The heat transfer from a rotating disc," Quart. J. Mech. Appl. Math. 17, 331-349.

[14] Smith, F. T., 1979, "On the non-parallel flow stability of the Blasius boundary layer," Proc. Roy. Soc. Lond. A 366, 91-109.

[15] Stewartson, K., 1964, "The theory of laminar boundary layers in compressible fluids," Oxford University Press.

[16] Stuart, J. T., 1971, "Nonlinear stability theory," Ann. Rev. Fluid Mech. 3, 347-370. 


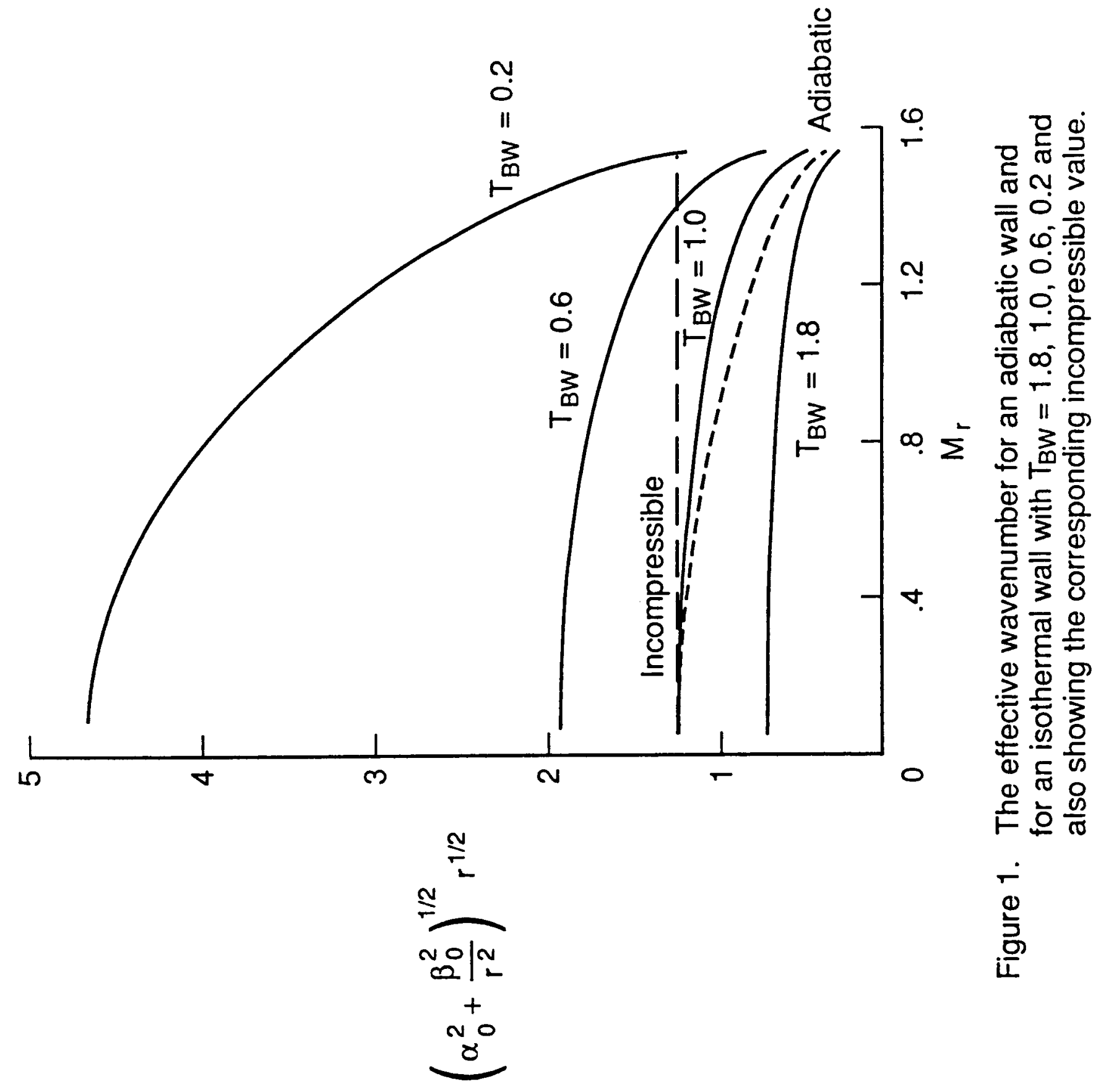




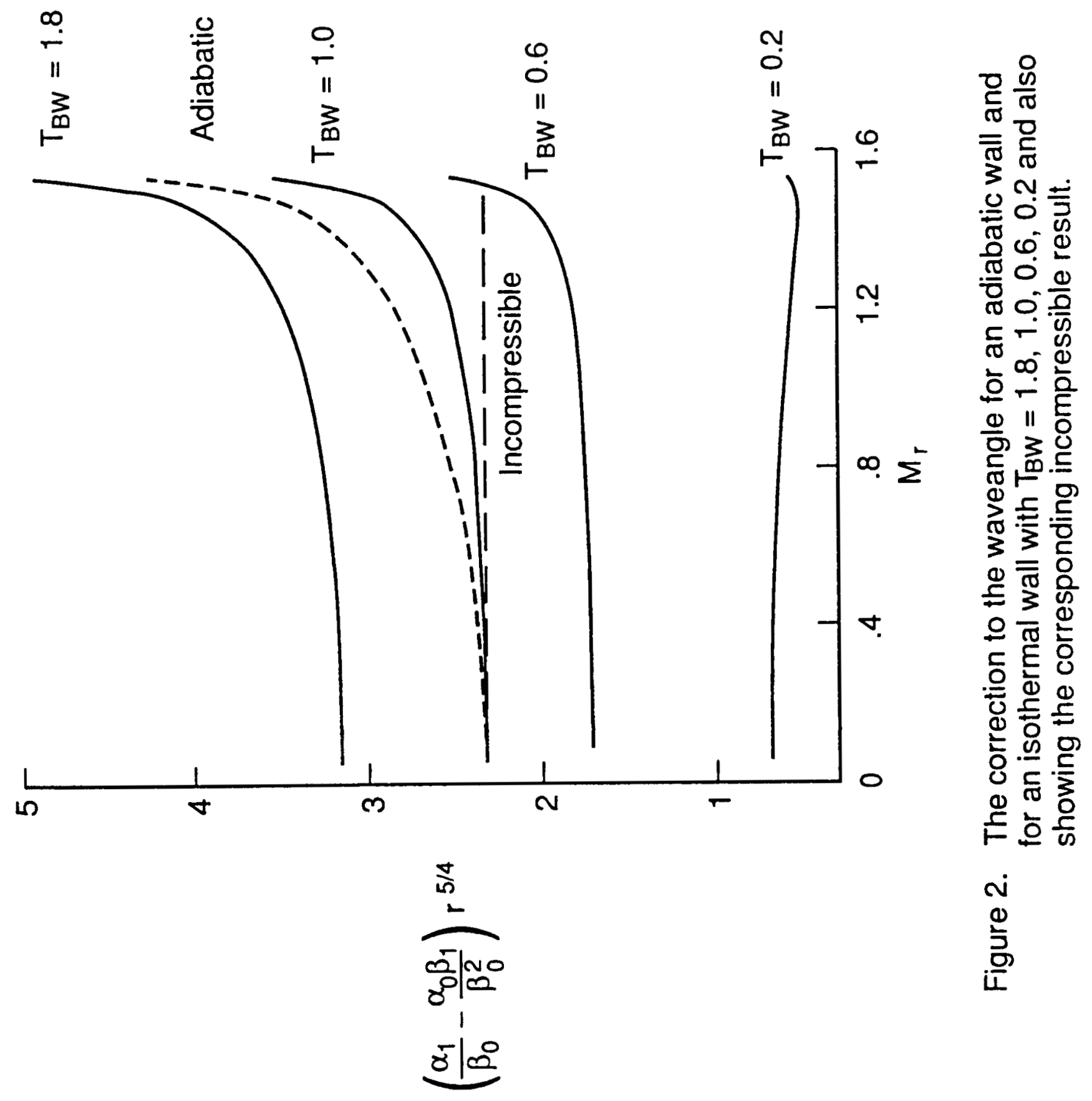




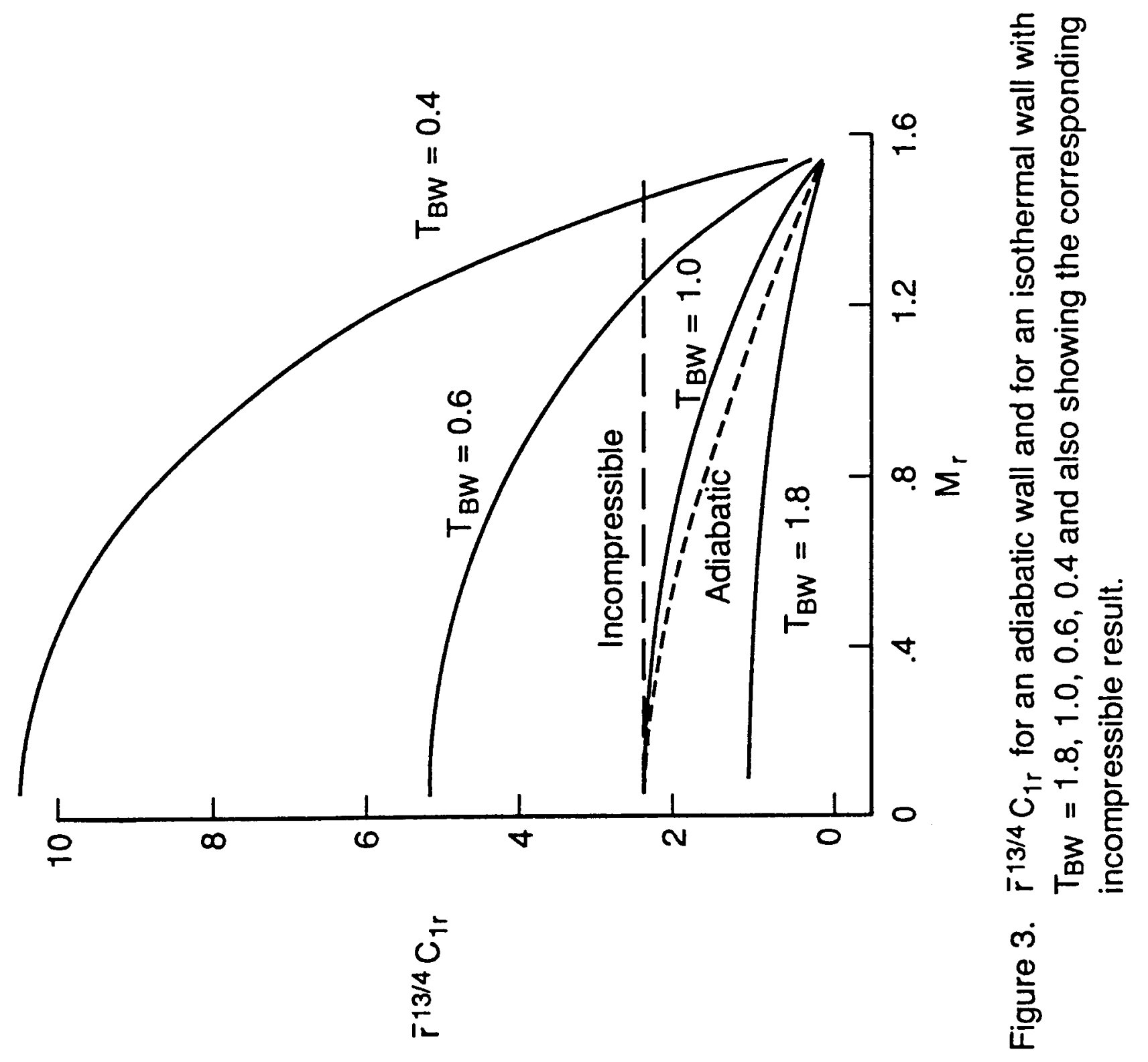




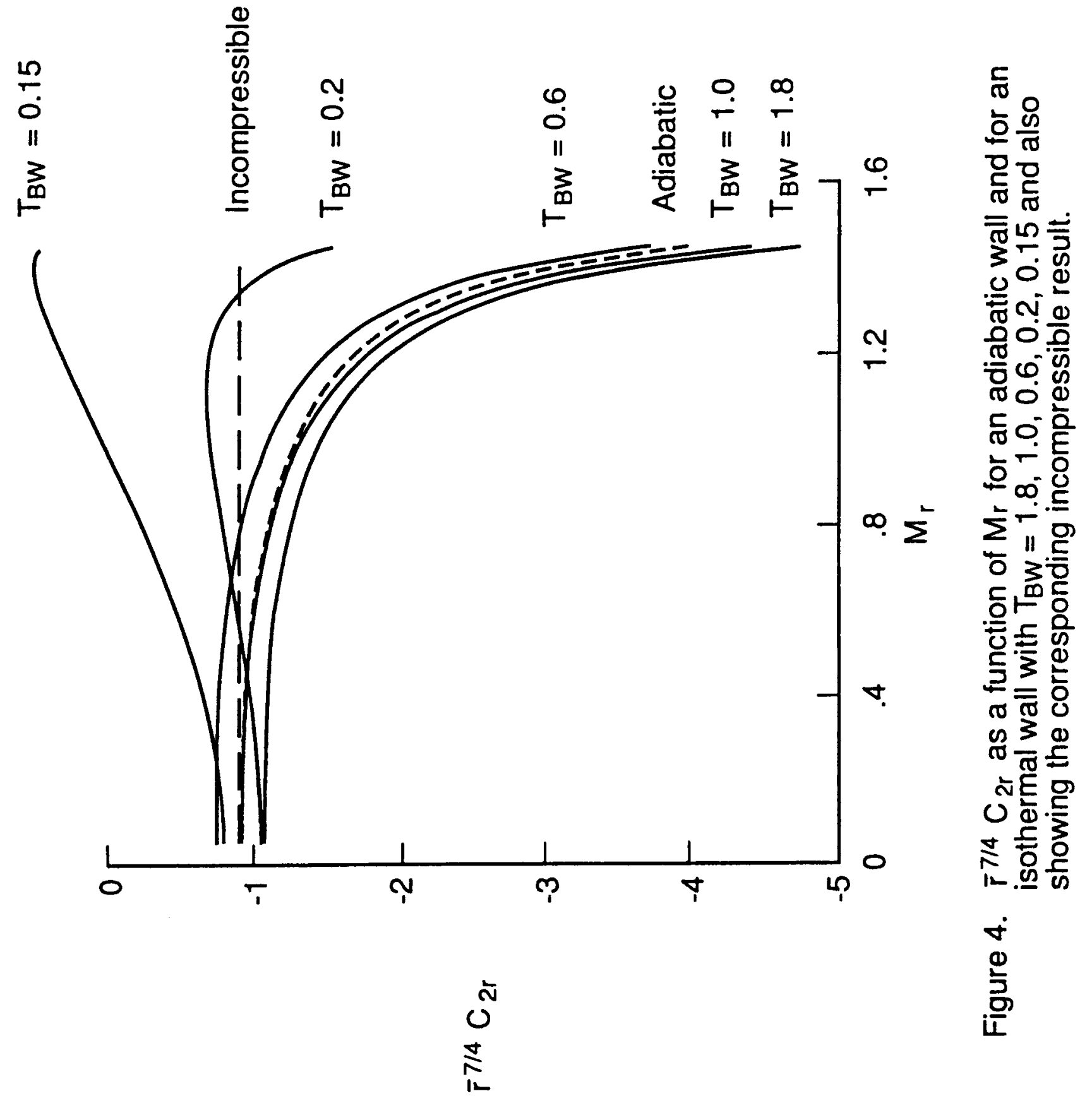




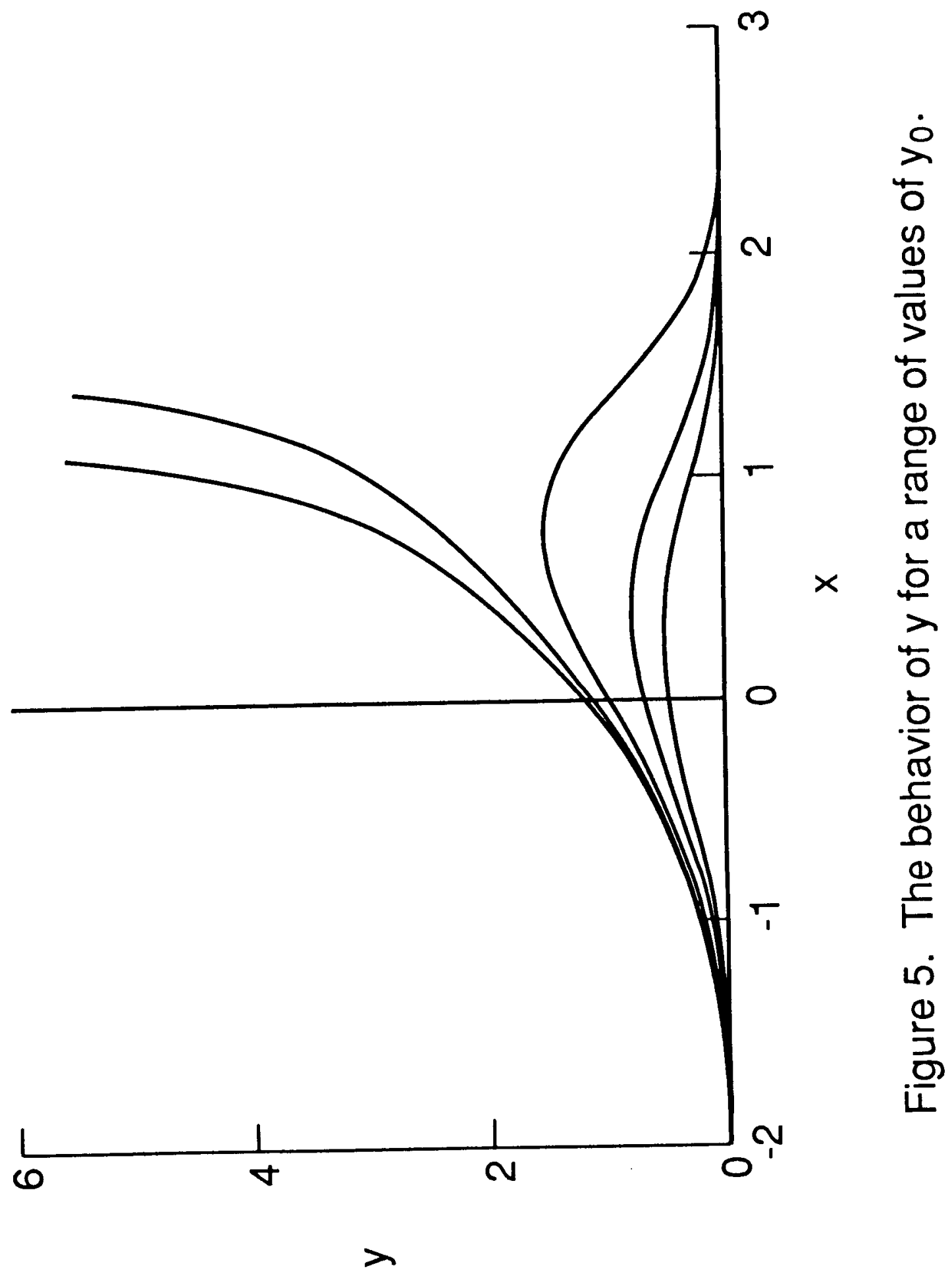




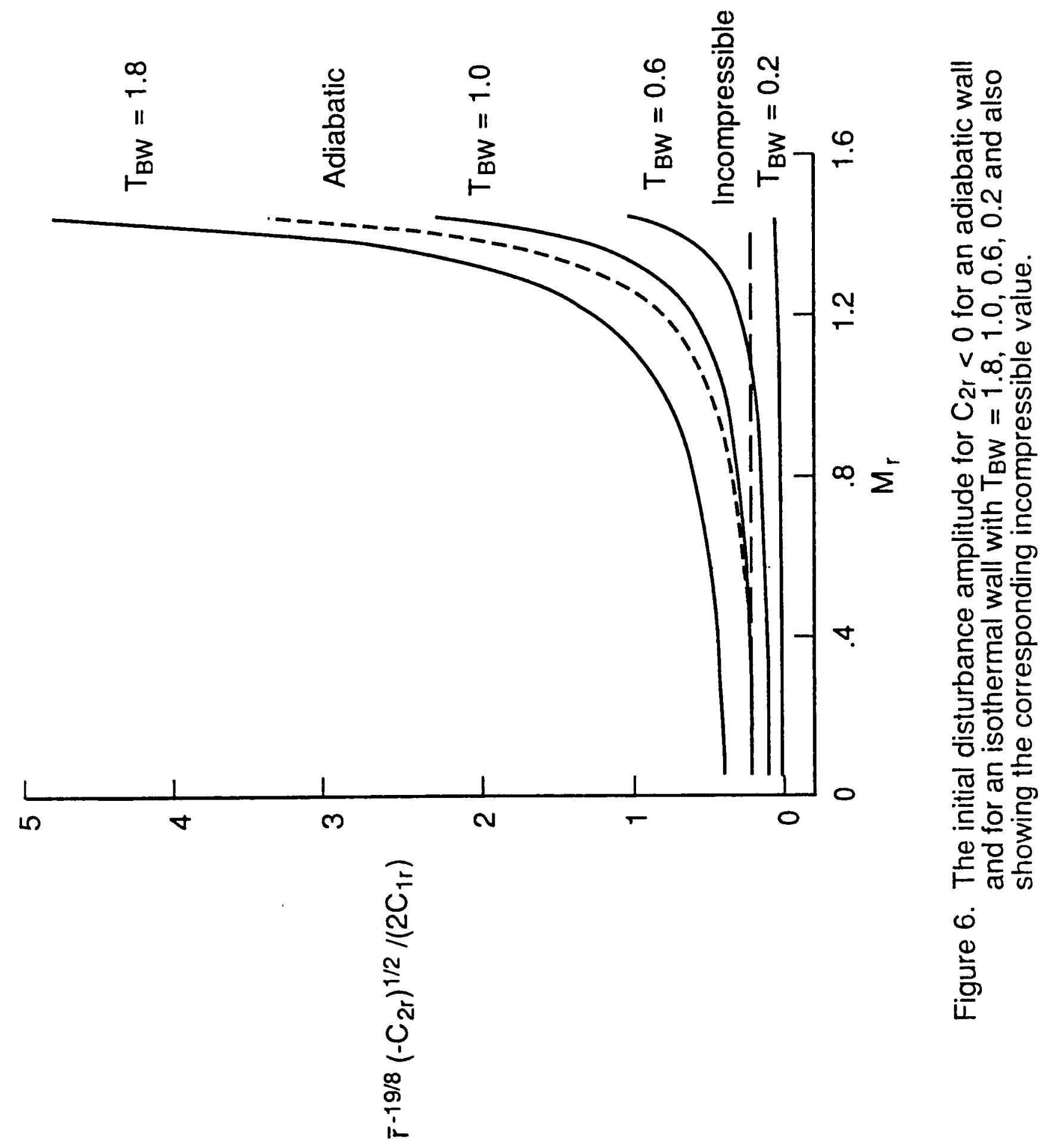


1. Report No.

NASA CR-181901

2. Government Accession No.

3. Recipient's Catalog No

ICASE Report No. $89-50$

4. Title and Subtitle

A NONLINEAR INVESTIGATION OF THE STATIONARY MODE OF INSTABILITY OF THE THREE-DIMENSIONAL COMPRESSIBLE BOUNDARY LAYER DUE TO A ROTATING DISC

7. Author(s)

S. o. Seddougui

$89-50$

10. Work Unit No.

9. Performing Organization Name and Address

Institute for Computer Applications in Science and Engineering

Mail Stop 132C, NASA Langley Research Center

Hampton, VA 23665-5225

12. Sponsoring Agency Name and Address

National Aeronautics and Space Administration

Langley Research Center

505-90-21-01

July 1989

6. Performing Organization Code

Hampton, VA 23665-5225

15. Supplementary Notes

Langley Technical Monitor:

Richard W. Barnwe11

The Quarterly Journal of Mechanics and Applied Mathematics

Final Report

16. Abstract

This work investigates the effects of compressibility on a stationary mode of instability of the three-dimensional boundary layer due to a rotating disc. The aim is to determine whether this mode will be important in the finite amplitude destabilization of the boundary layer. This stationary mode is characterized by the effective velocity profile having zero shear stress at the wall.! Triple-deck solutions are presented for an adiabatic wall and an isothermal wall. It is found that this stationary mode is only possible over a finite range of Mach numbers. Asymptotic solutions are obtained which describe the structure of the wavenumber and the orientation of these mdoes as functions of the lcoal Mach number. The effects of nonlinearity are investigated allowing the finite amplitude growth of a disturbance close to the neutral location to be described. The results are compared with the incompressible results of P. Hall (Proc. R. Soc. Lond. A406, 93-106 (1986)) and S. 0. MacKerrell (Proc. R. Soc. Lond. A413, 497-513 (1987)).

17. Key Words (Suggested by Author(s))

rotating disc, compressible boundary

layer, instability
18. Distribution Statement

34 - Fluid Mechanics and Heat Transfer 02 - Aerodynamics

Unclassified - Unlimited
20. Security Classif. (of this page)

Unclassified
Unclassified
22. Price

30

$\mathrm{A} 03$ 